\title{
Human Glial Progenitor Cells Effectively Remyelinate the Demyelinated Adult Brain
}

Windrem, Martha S.; Schanz, Steven J.; Zou, Lisa; Chandler-Militello, Devin; Kuypers, Nicholas J.; Nedergaard, Maiken; Lu, Yuan; Mariani, John N.; Goldman, Steven A.

Published in:

Cell Reports

DOI:

10.1016/j.celrep.2020.107658

Publication date:

2020

Document version

Publisher's PDF, also known as Version of record

Document license:

CC BY

Citation for published version (APA):

Windrem, M. S., Schanz, S. J., Zou, L., Chandler-Militello, D., Kuypers, N. J., Nedergaard, M., Lu, Y., Mariani, J. N., \& Goldman, S. A. (2020). Human Glial Progenitor Cells Effectively Remyelinate the Demyelinated Adult Brain. Cell Reports, 31(7), [107658]. https://doi.org/10.1016/j.celrep.2020.107658 


\section{Cell Reports}

\section{Human Glial Progenitor Cells Effectively Remyelinate the Demyelinated Adult Brain}

\section{Graphical Abstract}

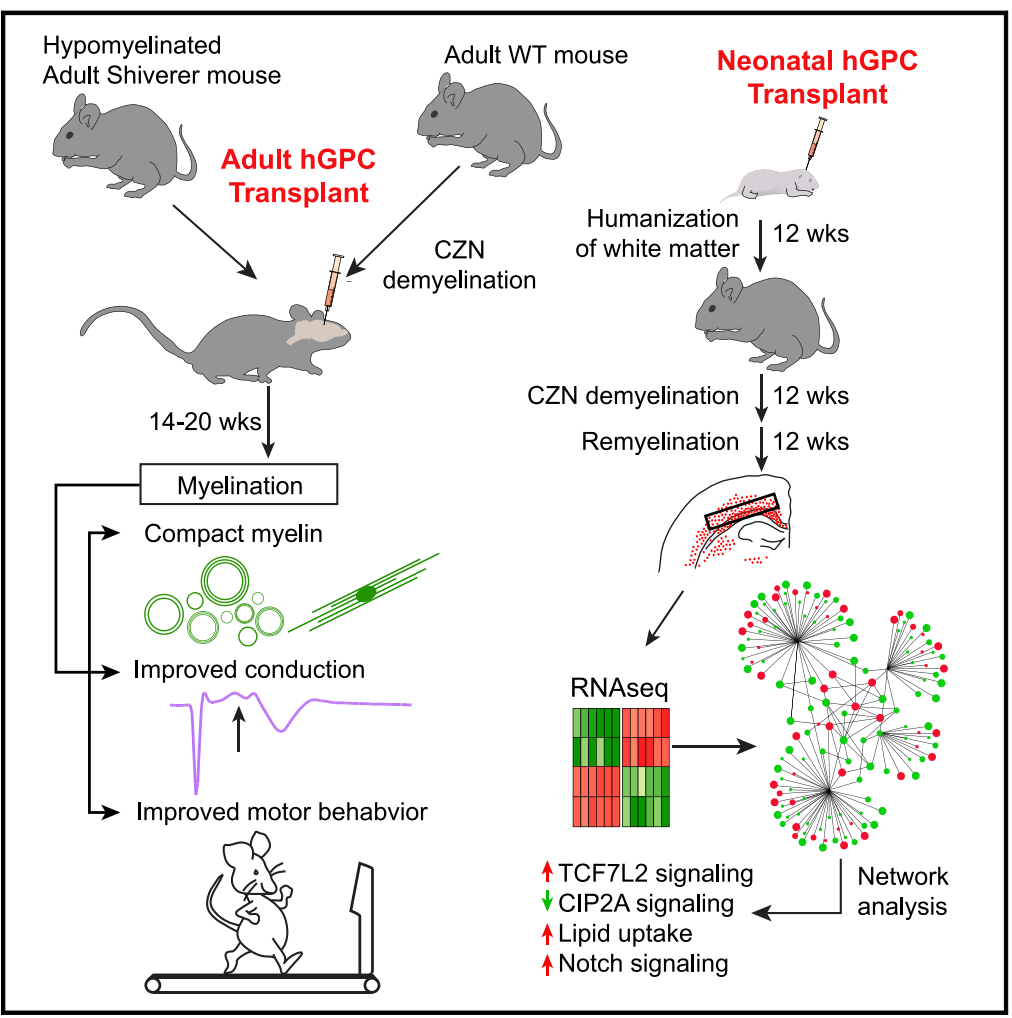

\section{Highlights}

- Transplanted human GPCs disperse to colonize the adult mouse forebrain

- Transplanted hGPCs myelinate the adult shiverer brain with functional improvement

- Transplanted hGPCs similarly remyelinate cuprizonedemyelinated brain

- RNA-seq of hGPCs reveals gene expression signature of human remyelination in vivo

\section{Authors}

Martha S. Windrem, Steven J. Schanz, Lisa Zou, ..., Yuan Lu, John N. Mariani, Steven A. Goldman

\section{Correspondence}

steven_goldman@urmc.rochester.edu

\section{In Brief}

Goldman and colleagues report that transplanted human glial progenitor cells can migrate broadly within the adult brain, colonizing the host to functionally remyelinate demyelinated tissue, in both congenital and acquired models of dysmyelination. They then use RNA-seq to define how the transcriptional signatures of human GPCs change after demyelination-induced mobilization. 


\title{
Human Glial Progenitor Cells Effectively Remyelinate the Demyelinated Adult Brain
}

\author{
Martha S. Windrem, ${ }^{1}$ Steven J. Schanz, ${ }^{1}$ Lisa Zou, ${ }^{1}$ Devin Chandler-Militello, ${ }^{1}$ Nicholas J. Kuypers, ${ }^{1}$ \\ Maiken Nedergaard, ${ }^{1,2}$ Yuan Lu, ${ }^{1}$ John N. Mariani, ${ }^{1}$ and Steven A. Goldman ${ }^{1,2,3,4, *}$ \\ ${ }^{1}$ Center for Translational Neuromedicine and the Department of Neurology, University of Rochester Medical Center, Rochester, NY 14642, \\ USA \\ ${ }^{2}$ Center for Translational Neuromedicine, University of Copenhagen, Copenhagen, Denmark \\ ${ }^{3}$ Neuroscience Center, Rigshospitalet, Copenhagen, Denmark \\ ${ }^{4}$ Lead Contact \\ *Correspondence: steven_goldman@urmc.rochester.edu \\ https://doi.org/10.1016/j.celrep.2020.107658
}

\section{SUMMARY}

Neonatally transplanted human glial progenitor cells (hGPCs) can myelinate the brains of myelin-deficient shiverer mice, rescuing their phenotype and survival. Yet, it has been unclear whether implanted hGPCs are similarly able to remyelinate the diffusely demyelinated adult CNS. We, therefore, ask if hGPCs could remyelinate both congenitally hypomyelinated adult shiverers and normal adult mice after cuprizone demyelination. In adult shiverers, hGPCs broadly disperse and differentiate as myelinating oligodendrocytes after subcortical injection, improving both host callosal conduction and ambulation. Implanted hGPCs similarly remyelinate denuded axons after cuprizone demyelination, whether delivered before or after demyelination. RNA sequencing (RNA-seq) of hGPCs back from cuprizone-demyelinated brains reveals their transcriptional activation of oligodendrocyte differentiation programs, while distinguishing them from hGPCs not previously exposed to demyelination. These data indicate the ability of transplanted hGPCs to disperse throughout the adult CNS, to broadly myelinate regions of dysmyelination, and also to be recruited as myelinogenic oligodendrocytes later in life, upon demyelination-associated demand.

\section{INTRODUCTION}

Oligodendrocytes are the sole source of myelin in the adult CNS, and their loss or dysfunction is at the heart of a wide variety of diseases of both children and adults. In children, the hereditary leukodystrophies accompany cerebral palsy as major sources of demyelination-associated neurological morbidity. In adults, demyelination contributes not only to diseases as diverse as multiple sclerosis and white matter stroke but also to a broad variety of neurodegenerative and neuropsychiatric disorders (Lee et al., 2012; Roy et al., 2004; Tkachev et al., 2003). As a result, the demyelinating diseases have evolved as especially attractive targets for cell-based therapeutic strategies (Archer et al., 1994, 1997; Brüstle et al., 1999; Kondo and Duncan, 2016; Shihabuddin et al., 2004; Tamaki et al., 2009; Windrem et al., 2002, 2004, 2008; Yandava et al., 1999; Zhang and Duncan, 2000), as reviewed in Franklin and Goldman (2015); Goldman et al., 2012, and Goldman (2016). Prior studies have established the ability of neonatally transplanted human glial progenitor cells (hGPCs) - also referred to interchangeably as oligodendrocyte progenitor cells (OPCs) or NG2 cells (Nishiyama et al., 2009)to myelinate the hypomyelinated shiverer brain and to rescue both the neurological phenotype and lifespan of mice treated as newborns (Wang et al., 2013; Windrem et al., 2004, 2008). Furthermore, a variety of enriched preparations of hGPCs, derived from both human brain and pluripotent stem cells, have been shown capable of remyelinating focal lesions of the adult brain and spinal cord (Buchet et al., 2011; Piao et al., 2015; Windrem et al., 2002). Yet, these latter studies were done in models of focal demyelination; it has remained unclear whether hGPCs are able to migrate extensively in adult brain tissue, as would be required for the repair of diffusely demyelinated tissue. Indeed, to our knowledge, no previous study has systematically assessed the ability of hGPCs to either migrate within or remyelinate brain tissue demyelinated in adulthood.

To that end, in this study, we asked if fetal-brain-derived hGPCs might exhibit sufficient migration and expansion competence in the adult environment to serve as therapeutic vectors for acquired, adult-onset demyelination. In this regard, recent studies have supported the readiness with which axons can remyelinate after either congenital or focal adult demyelination if provided myelinogenic cells (Buchet et al., 2011; Duncan et al., 2009; Ehrlich et al., 2017; Piao et al., 2015; Windrem et al., 2008). In parallel, other recent reports have highlighted the cell-intrinsic loss of myelination competence by endogenous glial progenitor cells as the basis for remyelination failure in disorders as diverse as progressive multiple sclerosis (Nicaise et al., 2017, 2019) and Huntington disease (Ferrari Bardile et al., 2019; Osipovitch et al., 2019), suggesting that the introduction of new, myelination-competent glial progenitors might be sufficient to 
achieve remyelination in those cases. On that basis, we asked here if hGPCs delivered directly into the adult brain could remyelinate axons in the setting of diffuse demyelination, as might be encountered clinically in progressive multiple sclerosis and other causes of multicentric adult demyelination.

To do so, we used three distinct experimental paradigms. We first asked if hGPCs could effectively disperse within and myelinate the brains of adult MBP ${ }^{\text {shi/shi }}$ mice, which carry the shiverer mutation in the gene encoding myelin basic protein (MBP); this mutation precludes MBP expression and abrogates developmental myelination in these mice. By these means, we assessed the ability of hGPCs to restore myelin to the congenitally hypomyelinated adult brain, as might be encountered in the late postnatal treatment of a hypomyelinating leukodystrophy. Second, we next asked if neonatally engrafted hGPCs could respond to cuprizone-induced adult demyelination by generating new oligodendrocytes and myelinating demyelinated axons, so as to assess the ability of already-resident hGPCs to remyelinate previously myelinated axons, as might be demanded of human parenchymal progenitor cells after acquired demyelination. Third, we then asked if hGPCs transplanted into the adult brain, after cuprizone demyelination, could remyelinate denuded axons, as might be anticipated in the cell-based treatment of disorders such as progressive multiple sclerosis.

We found that in each of these experimental paradigms, the hGPCs, whether engrafted neonatally or transplanted into adults, effectively dispersed throughout the forebrains, differentiated as oligodendroglia and myelinated demyelinated axons. These data suggested that transplanted hGPCs are competent to disperse broadly and differentiate as myelinogenic cells in the adult brain and, most critically, that they are able to remyelinate previously myelinated axons that have experienced myelin loss. On that basis, we also asked what the transcriptional concomitants of demyelination-associated mobilization might be in resident hGPCs. We, therefore, isolated hGPCs from neonatally chimerized brains after the cessation of cuprizone demyelination, and used RNA sequencing (RNA-seq) analysis to define those genes and cognate pathways induced by antecedent cuprizone demyelination. Together, these studies establish the ability of hGPCs to remyelinate demyelinated lesions of the adult human brain and provide a promising set of molecular targets for the modulation of this process in human cells.

\section{RESULTS}

Adult shiverer Mice Exhibit Myelination following hGPC Delivery

To assess the ability of donor hGPCs to disperse and differentiate as oligodendroglia in the adult brain, we introduced CD140a-sorted fetal hGPCs into young adult shiverer $\times$ rag2 $^{-1-}$ immune-deficient mice (Sim et al., 2011), as well as into two normally myelinated immunodeficient control lines, namely, rag ${ }^{-1-}$ on a C57BL/ 6 background and rag $2^{-/-}$on $\mathrm{C} 3 \mathrm{H}$. All mice were injected after weaning, over the range of 4-12 weeks of age; the shiverers were all injected between 4-6 weeks. A total of 22 mice (8 shiverers and 14 normally myelinated rag null mice, both rag $^{-1-}$ and rag $^{-/-}$) were injected bilaterally in the corpus callosum (CC), with injections of $5 \times 10^{4} \mathrm{hGPCs}$ per hemisphere.
All 8 shiverers and 6 of the controls were sacrificed $12-15$ weeks later at 19-22 weeks of age, and the remaining 8 control mice were sacrificed at approximately 1 year of age. The brains of all mice were examined for donor cell dispersal and oligodendrocytic differentiation as well as for MBP immunoreactivity, which was necessarily donor derived in the shiverer context.

The hGPCs were both highly migratory and robustly myelinogenic in the adult brain. By 12-15 weeks after transplant, the injected cells had dispersed broadly throughout the forebrain, as is typically observed in similarly treated neonates (Windrem et al., 2008), with a near-uniform distribution of donor cells noted throughout the white matter in both congenitally dysmyelinated shiverers (Figure 1A) and normally myelinated (Figure 1B) mice. Myelinogenesis was robust in the shiverers, with dense myelination of the corpus callosum (Figures 1C-1F). Importantly, at the 19-week time-point assessed, the callosal densities of all human cells, as well as hGPCs, oligodendrocytes, and astrocytes, were all significantly and substantially higher in the recipient shiverer brains than in their myelin wild-type (WT) controls (Figures 1G$1 \mathrm{M})$, indicating the overwhelmingly competitive advantage of the human donor cells in the shiverer environment. In myelin WT control brains, whether examined at either 5 or 12 months, these cells also expanded and engrafted but largely remained as progenitors (Figure $1 \mathrm{H}$ ). These data indicated that CD140asorted hGPCs are able to migrate broadly throughout the young adult mouse brain, that the dispersal of these cells is not impeded by adult brain parenchyma, and that robust myelination of still-viable axons can begin even after a several months' absence of mature myelin in the affected brain.

\section{Resident hGPCs Can Remyelinate the Cuprizone- Demyelinated Corpus Callosum}

Cuprizone is a well-studied copper chelator, the chronic oral administration of which causes mitochondrial dysfunction that is both earliest and most prominent in myelinating oligodendrocytes (Morell et al., 1998). Its oral administration results in diffuse, relatively synchronous demyelination, which has been well-characterized in a variety of mouse strains and ages (Stidworthy et al., 2003). Cuprizone-induced demyelination is more reproducible than any other current model of demyelination, has little systemic toxicity at demyelinating doses, is associated with little acute axonal injury or neuronal loss, and is relatively non-inflammatory, except for local microglial activation (Matsushima and Morell, 2001). To assess the ability of hGPCs to remyelinate newly demyelinated adult axons, we used dietary cuprizone to induce central demyelination and followed the responses of both already-resident and later-introduced hGPCs to that myelin loss.

We began by asking if hGPCs already resident within the mouse white matter could differentiate as oligodendrocytes and remyelinate denuded axons after cuprizone challenge. We first assessed the effects of cuprizone on rag $1^{-1-} \times$ C57BL/6 mice and confirmed previous observations (Hibbits et al., 2009, 2012) that a 12-week course of cuprizone induced the widespread loss of transferrin (TF)-defined oligodendrocytes in the corpus callosum, with no detectable loss of resident mouse GPCs. We then asked if neonatally implanted hGPCs could similarly tolerate cuprizone exposure and, if so, whether they 
A

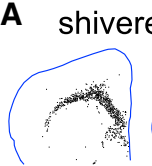
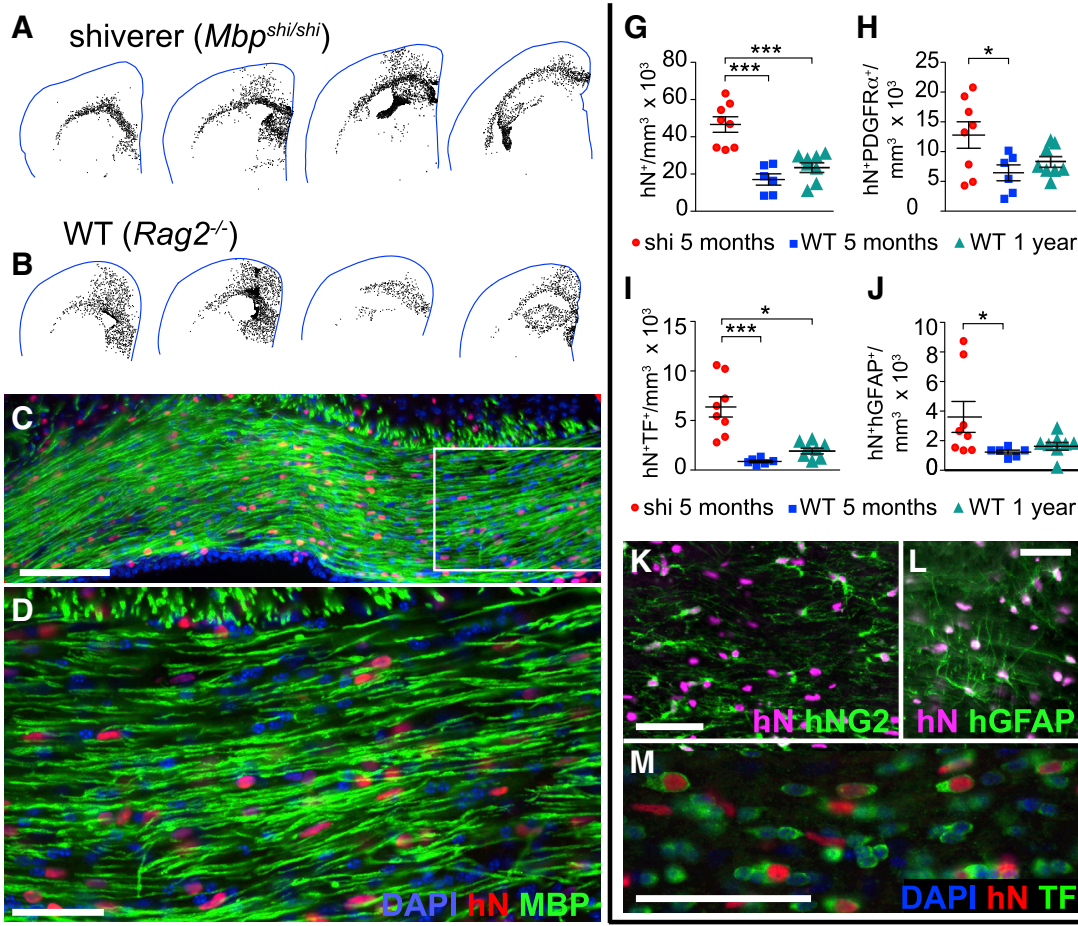

- shi 5 months $=$ WT 5 months $\triangle$ WT 1 year

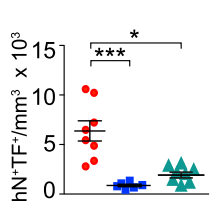

J

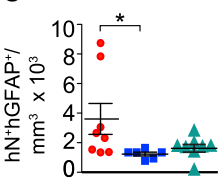

- shi 5 months -WT 5 months $\triangle$ WT 1 year
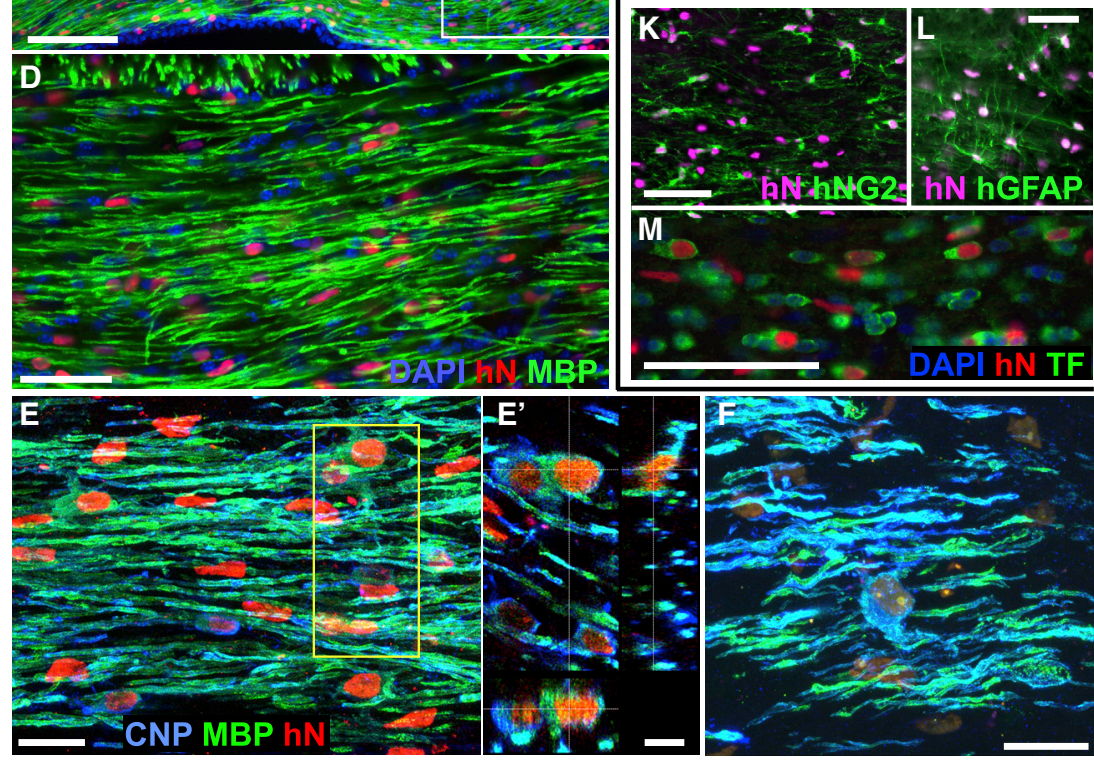

Figure 1. hGPCs Mediate Robust Myelination after Transplantation into the Adult shiverer Brain

hGPCs proved both highly migratory and robustly myelinogenic, after delivery at $4-6$ weeks of age to the hypomyelinated adult shiverer $\left(M B P^{s h i / s h i}\right) \times$ rag $^{-1}$ brain.

(A) By 19-20 weeks of age-13-15 weeks after transplant-the injected cells had dispersed as broadly as is typically observed in similarly transplanted shiverer neonates (Windrem et al., 2008, 2014), with a near-uniform distribution of donor cells noted throughout the forebrain white matter.

(B) hGPCs delivered to myelin wild-type (WT) rag2 ${ }^{-1}$ mice distributed throughout both gray and white matter, although with less mitotic expansion than that noted in hypomyelinated shiverer recipients.

(C) Oligodendrocyte differentiation and myelinogenesis by donor hGPCs delivered to the shiverer $x$ rag $^{-1-}$ brain at 6 weeks of age was robust, with dense myelination of the corpus callosum and fimbria by $19-20$ weeks.

(D) A higher power image of (C) shows the high proportion of donor cells in the now humanized host white matter.

(E) Confocal imaging through the callosum of an adult engrafted shiverer shows a dense array of myelinating human oligodendrocytes. (E') A higher-power field with orthogonal views.

(F) A single, oligodendrocyte myelinating multiple local axons in its vicinity. CNP, blue; MBP, green; $h N$, red. ( $G$ and H) Both shiverer (shi) and myelin WT recipients exhibited substantial donor hGPC colonization after adult transplantation, whereas the callosal densities of all human cells $(\mathrm{G})$ and PDGFaR-defined hGPCs $(\mathrm{H})$ were significantly higher in shiverer rather than WT recipients. In the latter, the densities of all donor cells and identified $\mathrm{hGPCs}(\mathrm{G}$ and $\mathrm{H})$ did not differ between 5 and 12 months of age, suggesting that donor cell expansion in the adult WT brain occurred within the first several months after transplant. ${ }^{*} p<0.05 ;{ }^{* \star *} p<0.001$

(I) The density of transferrin (TF)-defined human oligodendroglia was 5 - to 10 -fold higher in adult-transplanted shiverers than in WT hosts, when both were assessed 3 months after graft, at 5 months of age. ${ }^{*} p<0.05$; ${ }^{* \star *} p<0.001$.

(J) A smaller proportion of the donor cell population matured as GFAP-defined astrocytes; these were significantly more abundant in the shiverer than in WT hosts. ${ }^{*} \mathrm{p}<0.05$.

(K-M) Representative images of anti-human NG2-defined donor-derived hGPCs (K), anti-human GFAP-defined astrocytes (L), and TF/human nuclear antigen (hNA) co-expressing donor-derived oligodendrocytes (M), in 19-week-old shiverer white matter, 13 weeks after transplant.

Scale bars: $100 \mu \mathrm{m}(\mathrm{C}$ and $\mathrm{D})$ and $50 \mu \mathrm{m}(\mathrm{I}-\mathrm{K})$.

remained able to generate new oligodendrocytes and remyelinate adult-demyelinated axons. To that end, we transplanted rag $1^{-1-} \times$ C57BL/ 6 mice on post-natal day 1 with human fetal

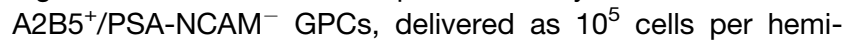
sphere into the corpus callosum bilaterally. For this study, we used $\mathrm{A}_{2} \mathrm{~B}^{+} / \mathrm{PSA}-\mathrm{NCAM}^{-}$-sorted GPCs rather than CD140adefined GPCs, in light of the cell numbers needed for this extensive experiment; the two antigenic phenotypes are lineally related and behave analogously in vivo in terms of both their migration and myelinogenic competence (Sim et al., 2011). This protocol results in widespread colonization of the recipient brains by hGPCs, which ultimately replace many-and typically most-of the host murine GPCs (Windrem et al., 2014). The resultant human glial chimeric mice were then given dietary cuprizone $(0.2 \% \mathrm{w} / \mathrm{w})$ as a food additive, beginning at 4 months of age; by this time, the $\mathrm{NG}^{+}$hGPCs have largely replaced mouse callosal NG2 ${ }^{+}$cells (Windrem et al., 2014). The experimental mice were left on the cuprizone diet for 12 weeks, and littermate controls were maintained on a normal diet (Figure 2A). The density of human cells in the host white matter, as well as the percentage of those cells that differentiated as oligodendrocytes, was calculated for both cuprizone-treated and control mice at the start of the dietary manipulation, as well as at each of 3 different time points thereafter: (1) immediately after cuprizone cessation, (2) after 8 weeks' recovery, and (3) after 20 weeks' recovery.

We found that the hGPCs tolerated cuprizone exposure at least as well as their mouse counterparts and dispersed broadly throughout the forebrain (Figures $2 \mathrm{~B}$ and $2 \mathrm{C}$ ). The hGPCs then robustly generated new oligodendrocytes and effectively 
A

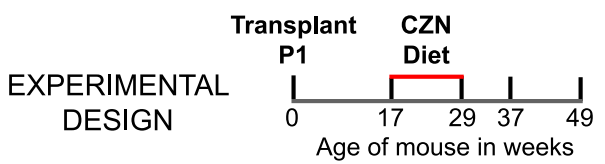

$\mathbf{B}$

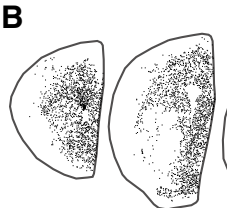

Control at 49 weeks

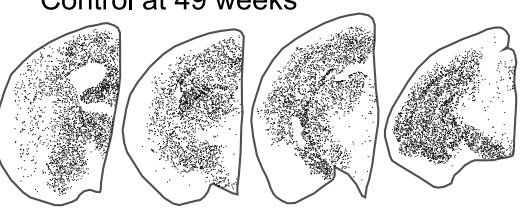

C

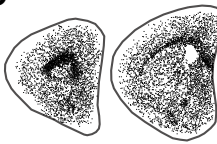

- Human cell

Cuprizone at 49 weeks

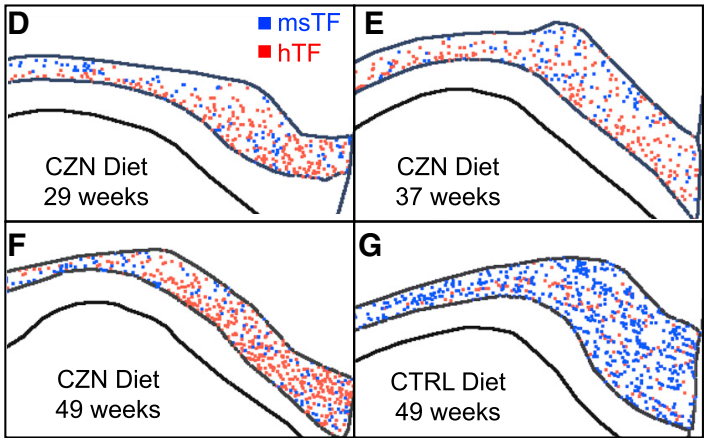

H

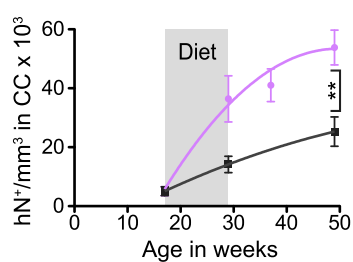

J

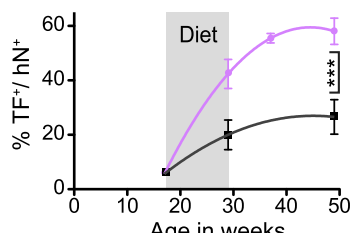

$\rightarrow$ Cuprizone Diet
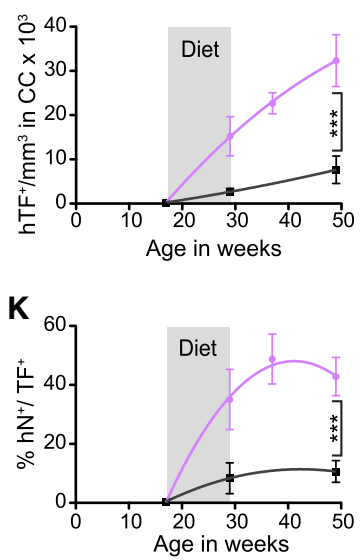

- Control Diet
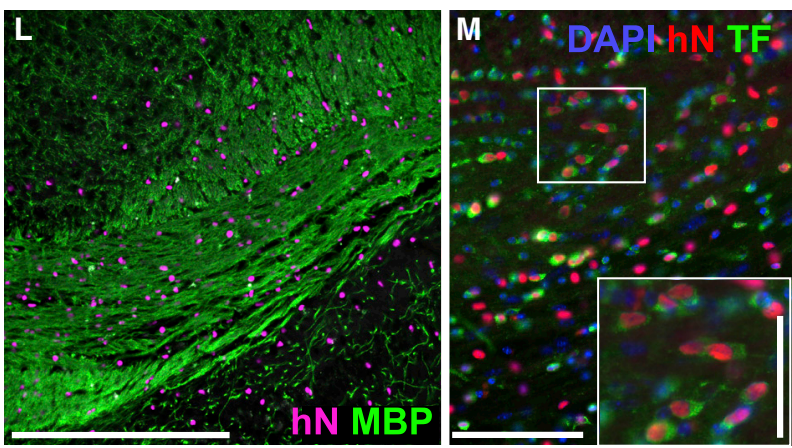

Figure 2. hGPCs Differentiate as Myelinogenic Oligodendroglia in Response to Cuprizone Demyelination

(A) The schematic outlines the experimental design for neonatal engraftment followed by adult demyelination. Mice were transplanted with $2 \times 10^{5}$ hGPCs perinatally, maintained on a control diet through 17 weeks of age, then placed on either a cuprizone-supplemented or normal diet for 12 weeks, then either sacrificed or returned to standard diet, and killed at later time points.

(B and C) Serial coronal sections comparing dot-mapped distributions of human (hNA) cells in control (B) and cuprizone-fed (C) mice at 49 weeks of age, following 20 weeks of recovery on the control diet.

(D-G) Relative positions and abundance of human (red dots) and mouse (blue) TF-defined oligodendrocytes, mapped in 20- $\mu \mathrm{m}$ coronal sections of corpus callosa of mice engrafted with hGPCs neonatally, demyelinated as adults from 17-29 weeks of age, and then assessed either at the end of the cuprizone diet (D), 8 weeks after return to control diet (E), or 20 weeks after cuprizone cessation (F). (G) Shows an untreated control, age-matched to (F).

(H) The density of human cells in the corpus callosum increases to a greater degree and more rapidly in cuprizone-demyelinated brains than in untreated controls, including during the 12-week period of cuprizone treatment (indicated in gray). ${ }^{* *} \mathrm{p}<0.001$.

(I) By 8 weeks after the termination of cuprizone exposure, the density of human oligodendroglia was $>5$-fold greater in cuprizone-demyelinated than in untreated control brains. ${ }^{\star \star \star} p=0.0006$.

( $\mathrm{J}$ and K) By that 80-week recovery point, over half of all hGPCs engrafted in the corpus callosa of cuprizone-treated mice had differentiated as oligodendrocytes $(\mathrm{J})$, and accordingly, over half of all TF-defined callosal oligodendrocytes were human (K); in contrast, relatively few human oligodendrocytes were noted in untreated chimeric brains. ${ }^{* *} \mathrm{p}<0.0001$.

(L) Substantial colonization by human glia evident in this remyelinated corpus callosum, after a 20-week recovery period (human nuclear antigen, magenta; myelin basic protein, green).

(M) Chimeric white matter populated, after cuprizone demyelination, by hGPC-derived oligodendroglia. hNA, red; TF, green; inset highlights relative abundance of $\mathrm{hNA}^{+} / \mathrm{TF}^{+}$human oligodendroglia. Scale bars: $100 \mu \mathrm{m}(\mathrm{L})$ and $50 \mu \mathrm{m}(\mathrm{M})$; inset scale bar: $25 \mu \mathrm{m}$.

remyelinated the demyelinated white matter after the cessation of cuprizone (Figures 2D-2G and 2I-2M). Both the total number of human cells and the percentage that differentiated as oligodendrocytes increased significantly faster and to a greater extent in the cuprizone-fed mice than in their matched controls (Figures $2 \mathrm{D}-2 \mathrm{G}$ and $2 \mathrm{H}-2 \mathrm{~K})$. The density of human cells in the corpus callosum of cuprizone-treated mice increased from 5,072 $\pm 1,611$ at 4 months to $53,835 \pm 5,898$ cells $/ \mathrm{mm}^{3}$ at one year; in contrast, over the same period, control mice exhibited a more modest expansion of GPCs to $25,296 \pm 4,959$ cells $/ \mathrm{mm}^{3}$ ( $p<0.001$ by 2-way ANOVA; F = 7.40) (Figure $2 \mathrm{H}$ ). Importantly, the proportion of all human cells that differentiated as TF-defined oligodendrocytes by 49 weeks was twice that in the cuprizone-treated mice compared with their untreated controls $(58.0 \% \pm 4.8 \%$ versus $26.6 \% \pm 6.4 \% ; p<0.0001, F=13.32$; Figure $2 \mathrm{~J}$ ). Accordingly, the terminal density of human oligodendrocytes in the 
A

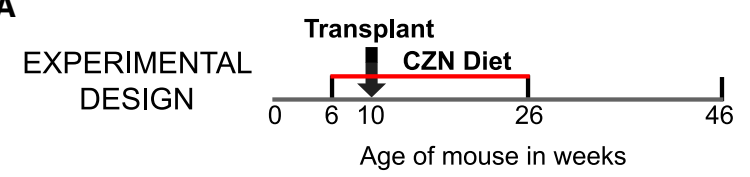

B
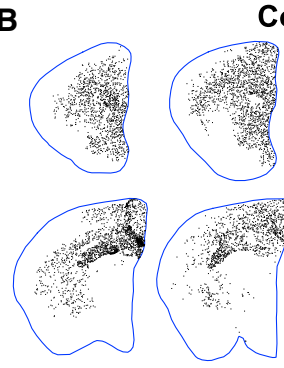

Control at 46 weeks
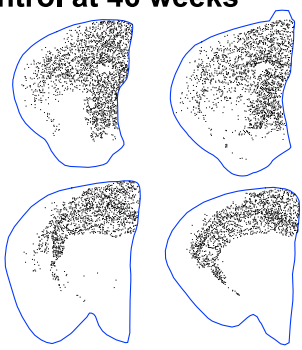

$\mathbf{C}$

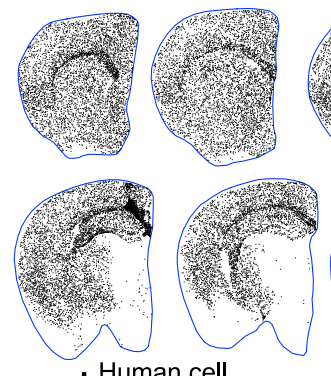

Cuprizone at 46 weeks
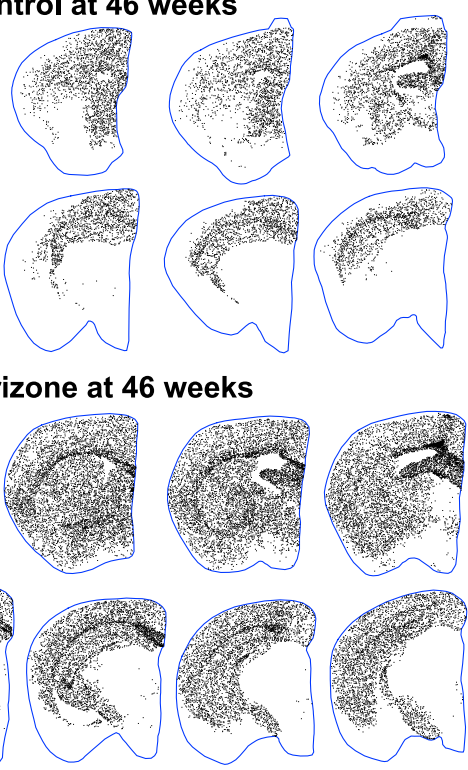

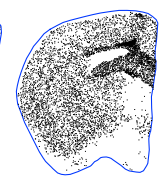

- Human cell

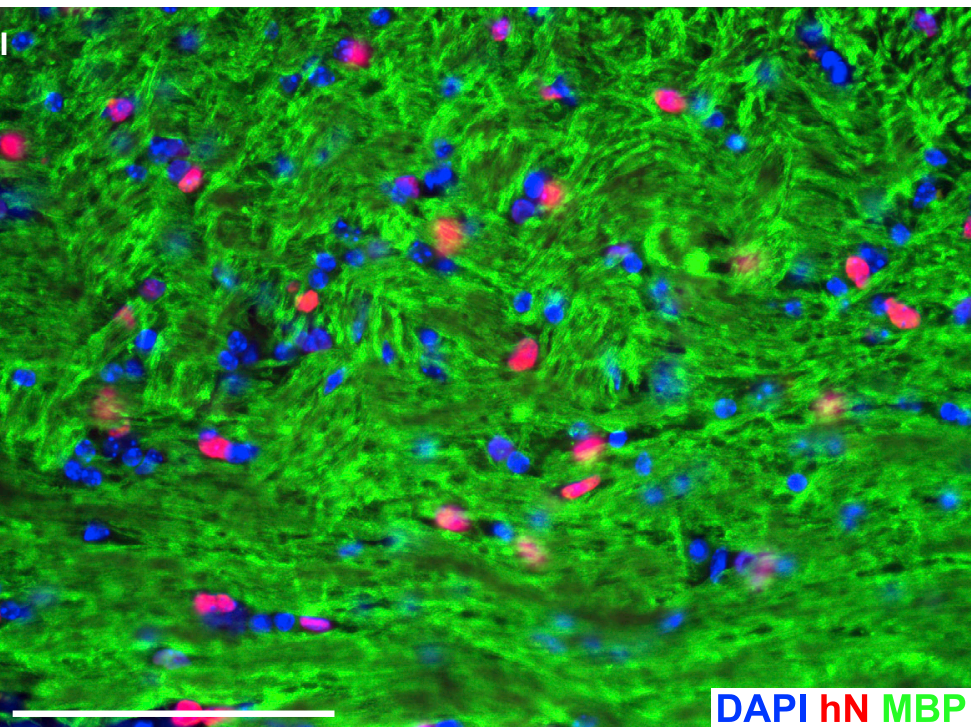

Cuprizone

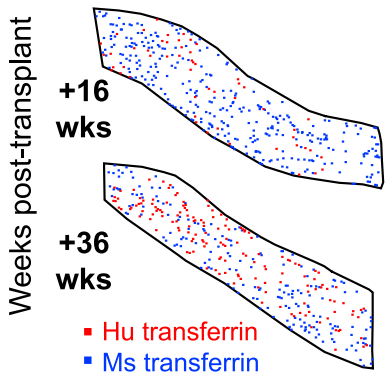

E

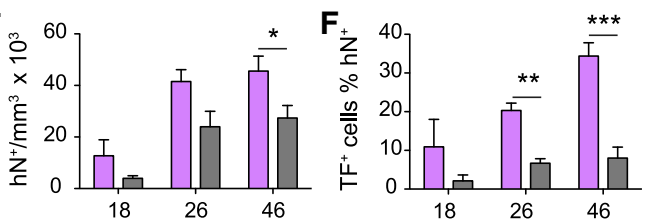

G

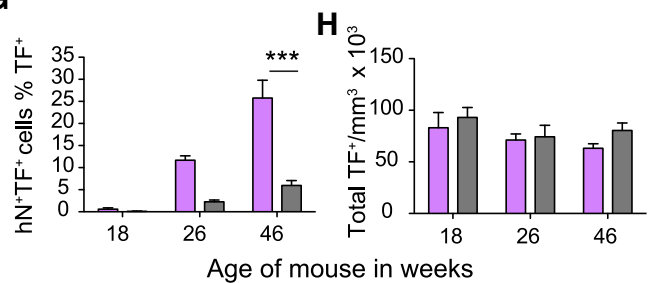

$\square$ CZN $\square$ CTRL

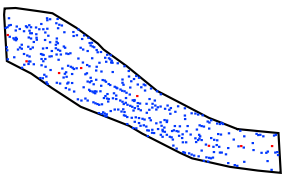

\section{Control}
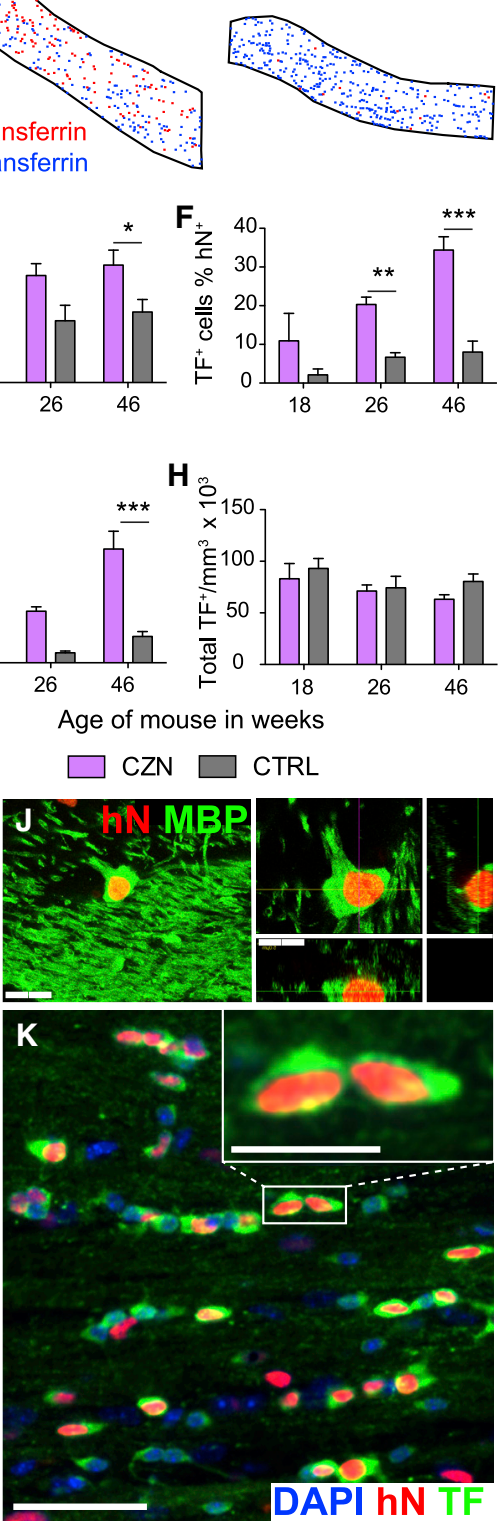

Figure 3. hGPCs Differentiate and Remyelinate Axons after Transplant into Adult-Demyelinated Brain

(A) At 6 weeks of age, experimental mice were put on a diet containing $0.2 \%$ cuprizone, whereas littermate controls remained on a standard diet. At 10 weeks, 4 weeks into a 20-week cuprizone course, the mice were transplanted with $2 \times 10^{5} \mathrm{hGPCs}$. Mice were sacrificed for histology either at the end of the cuprizone course (at 26 weeks) or after an additional 20-week recovery period (at 46 weeks).

(B and C) Maps show locations of individual human cells in 20- $\mu$ m coronal hemi-sections of engrafted brains. (B) Transplantation of hGPCs into a normally myelinated 10-week-old mouse yielded widespread engraftment, when mapped 36 weeks later at 46 weeks of age. (C) In cuprizone-treated mice, transplanted hGPCs expanded to a significantly greater degree.

(D-H) Significantly more hGPCs differentiated as TF-defined oligodendrocytes in the cuprizone-demyelinated brains than in their untreated controls. (D) hGPCs were more likely to differentiate as TF-expressing oligodendrocytes when transplanted into a demyelinating environment (left) than into a control brain (right). $(E$ and $F)$ The absolute density of human cells $(E)$ and relative proportion that differentiated as $\mathrm{TF}^{+}$oligodendrocytes $(\mathrm{F})$ in the corpus callosum were $>5-$ and 
cuprizone-treated chimeric mice was 4.5-fold greater than that of untreated chimeric mice $(32,323 \pm 5,850$ versus $7,642 \pm 3,095$ $\mathrm{hTF}^{+}$cells $/ \mathrm{mm}^{3}$ at 49 weeks; $\mathrm{p}=0.0006, \mathrm{~F}=8.65$; Figure 2l). For these latter measurements, we immunostained for TF, which is cytoplasmic and membrane localized, in tandem with human nuclear antigen, so as to quantify and distinguish oligodendrocyte numbers by the species of origin (Connor and Fine, 1987; Connor et al., 1993).

These data indicate that cuprizone-induced demyelination yielded a relative increase in both the absolute numbers and relative proportions of parenchymal hGPC-derived oligodendrocytes that, in turn, mediated the remyelination of denuded host axons in these animals (Figures $2 \mathrm{~L}$ and $2 \mathrm{M}$ ). Thus, those hGPCs already resident within the callosal white matter responded to acute demyelination by expanding, with a fraction of their daughters differentiating as mature oligodendrocytes and remyelinating accessible axons. As such, these data indicate that hGPCs can myelinate not only axons that have never been myelinated, as in the adult shiverer, but also axons that have been previously ensheathed by myelin.

\section{hGPCs Can Remyelinate Axons when Delivered after Initial Demyelination}

We next asked if hGPCs delivered to the adult brain after initial demyelination and during ongoing cuprizone exposure could migrate and myelinate host axons and whether they were able to myelinate denuded axons as effectively as hGPCs resident in their host brains since neonatal development. To this end, we next used prolonged cuprizone exposure to demyelinate otherwise WT adult mice and transplanted hGPCs into these demyelinating brains. In particular, to minimize the potential for endogenous remyelination by remaining mouse GPCs, we used a 20-week cuprizone course, which we found allowed much less spontaneous remyelination than shorter periods of cuprizone exposure (Figure 3A). We found that even when delivered into adult brain parenchyma 4 weeks after the onset of cuprizone treatment during active demyelination, the transplanted hGPCs not only dispersed widely but also expanded more robustly than in untreated controls (Figures 3B-3E). When the cuprizone-fed mice were assessed at 16 weeks after hGPC transplant (26 weeks of age), human oligodendrocytes were apparent, having differentiated from the engrafted hGPCs. By that point, more donor hGPCs had differentiated as oligodendrocytes in the cuprizone-demyelinated brains than in their untreated controls, suggesting both the preferential expansion of hGPCs (Figure 3E) and the active induction of oligodendrocytic phenotype by the demyelinated environment (Figure 3F). By 36 weeks post-transplant (46 weeks of age), after allowing 20 additional weeks for phenotypic differentiation, over a quarter of all oligodendrocytes in the host white matter were of human origin (Figure 3G). Remarkably, the overall number and density of TF-defined oligodendrocytes, whether of mouse or human origin, were relatively preserved at all time points (Figure $3 \mathrm{H}$ ). The transplanted human cells proceeded to robustly differentiate as oligodendrocytes and myelinate the demyelinated tissue, such that by 46 weeks of age-36 weeks post-transplantmuch if not most of the forebrain white matter in these previously cuprizone-demyelinated brains was of human origin (Figures 31$3 K)$. Thus, hGPCs were able to effectively remyelinate mature axons that had been previously myelinated in the brain and could do so even when delivered to the adult brain after the onset of demyelination.

\section{Progenitor-Mediated Myelination Was Associated with} Functional Improvement

To assess the functional correlates of hGPC-mediated remyelination, we focused on adult-transplanted shiverer mice as the more extreme and functionally impaired of the models we assessed. To this end, a cohort of shiverer $\times$ rag2 $2^{-1-}$ mice were transplanted between 4-6 weeks of age with a total of $4 \times 10^{5}$ hGPCs, delivered as divided doses to the corpus callosum and striatum bilaterally; a matched cohort of shiverers was sham injected. The mice were then serially assessed at 18-20 weeks for the following (Figure 4A): (1) motor function, as measured by DigiGait automated treadmill analysis; (2) transcallosal conduction amplitudes and velocities, relative to the sham-operated shiverer controls, as determined by their stimulation-induced compound action potentials in slice preparations; and (3) confocal and electron microscopic analyses of donor-derived myelin.

The DigiGait analysis (Figures $4 \mathrm{~B}$ and $4 \mathrm{C}$ ) revealed that by 18 weeks, the motor function and ambulation of transplanted shiverer mice had improved overtly relative to untransplanted controls: among a sample of 8 treated and 8 control mice, only 2 of the 8 untreated controls were able to remain on the moving treadmill for the minimal 5-s testing period, whereas 7 of the 8 transplanted shiverers were able to do so on their first try, and all 8 thereafter, with sustained treadmill-elicited ambulation at $10 \mathrm{~m} / \mathrm{s}(\mathrm{p}=0.012$; chi-square $=6.35,1$ degree of freedom $[\mathrm{df}$; $Z=2.52$ ) (Figure 4C; Video S1).

These transplant-associated improvements in shiverer motor performance were associated with normalization, relative to WT mice, in the amplitude of the N1 (large myelinated fiber) component of the transcallosal compound action potential (Figures 4D-4F), as assessed in slice preparations of geographically homologous coronal sections of transplanted and untransplanted 18- to 19-week-old shiverers ( $n=4$ or 5 mice/group). Indeed, sham-injected shiverer mice did not have a detectable $\mathrm{N} 1$, whereas their transplanted counterparts did so reliably (Figure $4 \mathrm{E}$ ). In addition, slices derived from the transplanted brains exhibited a significant increase in the mean velocity of callosal

$>10$-fold greater, respectively, in mice on the cuprizone diet than in their untreated controls. (G) By 36 weeks post-transplant, over a quarter of all oligodendrocytes in the host white matter were of human origin. $(H)$ The overall density of TF-defined oligodendrocytes, whether of mouse or human origin, was relatively preserved at all time points. (E-G) ${ }^{\star} p<0.05 ;{ }^{\star \star} p<0.01 ;{ }^{\star \star \star} p<0.001$.

(I-K) By 46 weeks, adult-transplanted hGPCs are admixed with murine cells in the largely remyelinated corpus callosum (I). (J) By this point, most myelinating oligodendrocytes in the cuprizone-demyelinated callosal were of human donor origin (human nuclear antigen, red; MBP, green; DAPI, blue), and just as many of the resident human cells had differentiated as TF-defined oligodendrocytes (K) (human nuclear antigen, red; TF, green).

Scale bars: $100 \mu \mathrm{m}(\mathrm{I}), 10 \mu \mathrm{m}(\mathrm{J})$, and $50 \mu \mathrm{m}(\mathrm{K})$. 
A

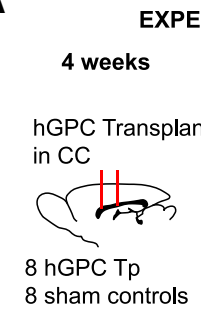

D

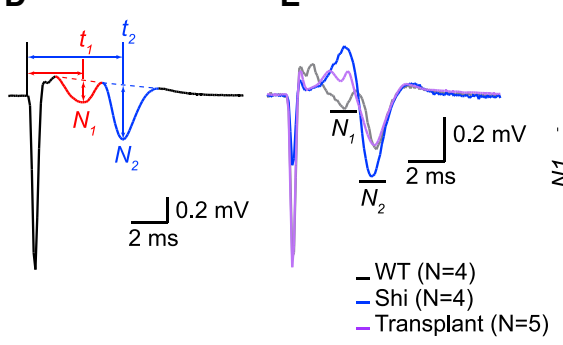

B

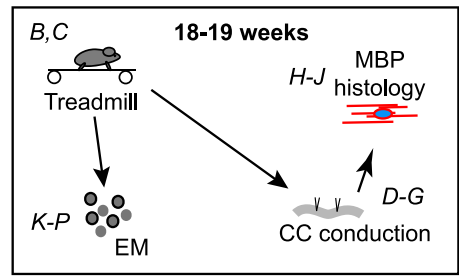

F

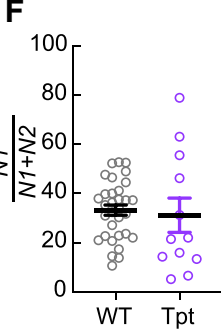

C
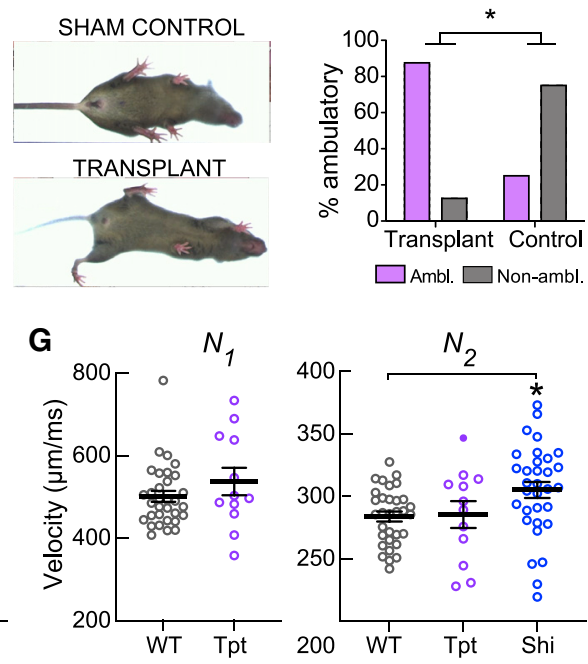
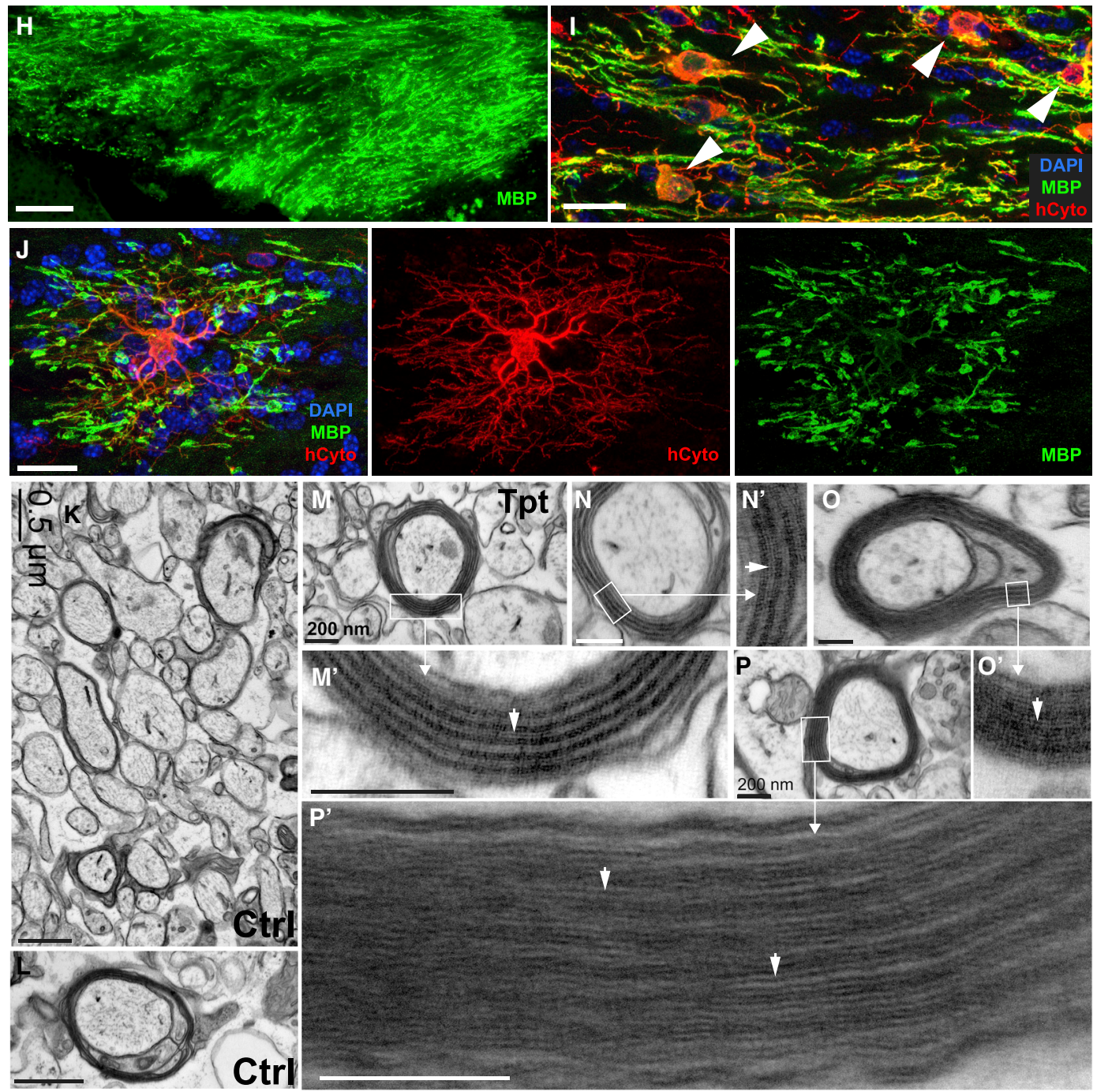
conduction, as reflected in the latency of the N2 component, whereas the untreated shiverers had an N2 velocity of $305.2 \pm$ $6.4 \mathrm{~m} / \mathrm{s}$, and slices derived from transplanted shiverers (with analysis restricted to those with a measurable N1 component) manifested a slower velocity to $\mathrm{N} 2$ of $285.5 \pm 10.7 \mathrm{~m} / \mathrm{s}$ ( $p=0.02$ by one-way ANOVA; $F=4.07,73 \mathrm{df}$ ), which was indistinguishable from that of WT myelin WT controls $(283.9 \pm$ $4.0 \mathrm{~m} / \mathrm{s}$ ) (Figure 4G).

These measures of functional improvement in the engrafted shiverers reflected the improved myelination of these transplanted animals. Confocal imaging confirmed the oligodendrocytic differentiation of, and axonal myelination by, the transplanted human donor cells (Figures $4 \mathrm{H}-4 \mathrm{~J}$ ). In addition, ultrastructural analysis confirmed that the hGPC-engrafted shiverers developed compact myelin by the time of sacrifice, 12-14 weeks after transplantation, with the formation of major dense lines (Figures 4M-4P), associated with and degrees of myelin ensheathment not observed in untransplanted shiverer controls (Figures 4K and 4L; compare to Figures 4M-4P).

\section{hGPCs Activated Stereotypic Transcriptional Programs after Cuprizone Demyelination}

We next asked whether demyelination and its attendant activation of hGPCs was associated with transcriptional events that might identify early determinants of progenitor cell mobilization, as well as those of astrocytic or oligodendrocytic fate. To thereby identify the responses of hGPCs to demyelination in vivo, we isolated them from cuprizone-demyelinated chimeric brains in which they had been resident since birth, by using CD140adirected fluorescence-activated cell sorting (FACS), followed by RNA-seq. To this end, neonatal rag $1^{-1-}$ mice were transplanted with fetal hGPCs and maintained through 12 weeks of age on a normal diet. At that point, control mice were continued on a normal diet, whereas the experimental mice were transitioned to a diet of $0.2 \%(\mathrm{w} / \mathrm{w})$ cuprizone for 12 weeks to induce oligodendrocytic death. The cuprizone-demyelinated mice were then allowed to recover for an additional 12 weeks on a normal diet, before both groups were sacrificed at 36 weeks of age. The callosal white matter was then dissected and dissociated, and CD140a ${ }^{+}$hGPCs were isolated by FACS. The RNA of these hGPC isolates was then extracted and sequenced.

Principal-component analysis of these normalized RNA-seq samples revealed tight clustering of control (CTR) samples, which as a group were readily distinguished from their post-cuprizone counterparts (Figure 5A). Both the post-cuprizone and CTR hGPCs were enriched for genes associated with early oligodendroglial lineage, including CNP, GPR17, NKX2-2, OLIG1, OLIG2, SOX10, CSPG4, and ST8SIA1, as well as the selection marker PDGFRA/CD140a, with the latter validating the selectivity and efficacy of the sort (Figure 5B). In contrast, the hGPC isolates exhibited low to undetectable expression of a number of neural stem cell, neural progenitor, endothelial, microglial, neuronal, and astrocytic markers. Yet, although both groups presented with transcriptional signatures consistent with hGPC phenotype, a total of 914 transcripts were differentially expressed between hGPCs following recovery from cuprizone treatment and their control counterparts (adjusted $p<0.05$ ). Of these, 777 genes were upregulated in cuprizone-treated GPCs, whereas 137 were downregulated (Table S1). Functional analysis of this gene set demonstrated that the cuprizone-treated hGPCs differentially expressed gene ontologies reflecting cell proliferation, pathfinding and cell movement, and the initiation of myelination (Figure 6; Table S2).

\section{Network Analysis Revealed That Cuprizone-Exposed hGPCs Were Primed to Oligoneogenesis}

To aid in interpreting these data (deposited to GEO: GSE112557), a post-cuprizone hGPC expression network was constructed based upon significantly enriched gene ontologies and differentially expressed individual gene components thereof. The network included 43 significantly enriched and relevant functional terms, in addition to their contributing differentially-expressed genes (network in Figure 5C; functionally segregated heatmaps in Figure 5D; gene ontologies and components

Figure 4. Myelination by Adult-Transplanted hGPCs Restores Function in shiverer Mice

(A) Mice were injected bilaterally in the corpus callosum and striatum at 4 weeks of age with either cells or vehicle. When 18 weeks old, the mice were examined sequentially in a series of tests spanning behavior to anatomy. The arrows indicate the sequence of tests, and italics indicate the relevant figures.

(B) Sham-injected (top) and hGPC-transplanted (bottom) shiverers were videoed from below on a treadmill by using DigiGait.

(C) Of the 8 shiverer controls and 8 transplanted shiverers tested on the treadmill, only 2 of 8 untreated mice were able to walk on the treadmill, whereas 7 of 8 engrafted mice did so on their first try $(p=0.012$, chi-square; see Video S1), and all 8 did so on a second attempt.

(D) Schematic of the measurements taken to assess the trans-callosal response to electrical stimulation of sampled slices.

(E) Representative traces of normally myelinated shiverer heterozygous mice (WT), sham-injected shiverers (sham), and transplanted shiverers (Tpts), demonstrating restoration of the fast conduction $\mathrm{N} 1$ component in the transplanted mice.

(F) N1 amplitude shows no significant difference between slice preparations derived from normal WT and transplanted shiverer (Tpt) brains, suggesting transplant-mediated normalization of the ratio of myelinated to unmyelinated axons in the engrafted shiverer brain. Untreated shiverers have effective N1 velocities of zero and cannot be assessed as such.

(G) The slow N2 component conduction velocity of transplanted shiverer callosal slices was significantly more rapid than that of untreated shiverers and no different from that of WTs.

$(\mathrm{H}-\mathrm{P})$ All images were taken from 18-week-old shiverermice mice injected intracallosally at 4 weeks with hGPCs. $(\mathrm{H})$ Low-magnification image of the corpus callosum (CC) of one hemisphere of an adult-engrafted shiverer. MBP, green. (I) Human oligodendrocytes, co-immunostained for human cytoplasmic antigen (hCyto) and MBP; the latter is necessarily human, as shiverer does not make MBP. (J) A single mature donor-derived oligodendrocyte. Right-hand images show respective color splits for human cytoplasm (red) and MBP (green), showing the many myelinating sheaths produced by a single hGPC-derived oligodendrocyte. $(\mathrm{K}-\mathrm{L})$ Electron micrographs of a sham-control CC demonstrating the thin myelin sheaths characteristic of shiverer. (M-P) Electron microscopy images of transplanted shiverers. (M-O) The variety of donor cell-ensheathed host axons, and the insets show higher power images of the major dense lines (arrowheads), characteristic of compact myelin. $(\mathrm{P})$ Higher power image of the detailed myelin ultrastrure of a donor cell-myelinated axon.

Scale bars: $100 \mu \mathrm{m}(\mathrm{H}), 20 \mu \mathrm{m}(\mathrm{I}-\mathrm{J}), 500 \mathrm{~nm}$ (K and L), $200 \mathrm{~nm}(\mathrm{M}-\mathrm{P})$, and $100 \mathrm{~nm}\left(\mathrm{M}^{\prime}-\mathrm{P}^{\prime}\right)$. 
A

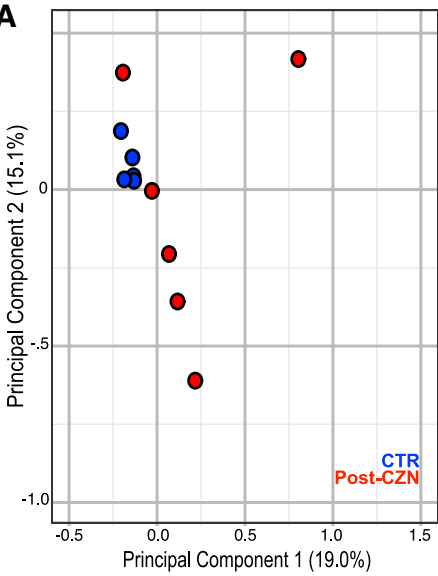

B

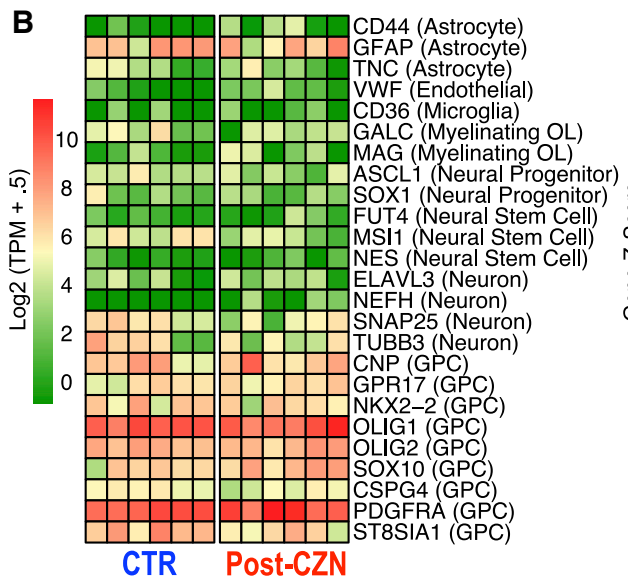

C Node Type:

Gene upregulated in Post-CZN hGPC

Gene downregulated in Post-CZN hGPC

$\triangle$ Gene Ontology Term

Node Degree

- 1

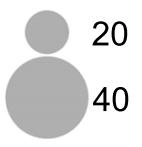

GO enrichment - $\log (p-$ value $)$

1

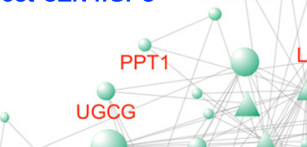

FGFRL1

M1 $=18.8 \%$

Myelination CNS Development
TCF7L2 Signaling UGCG 100 PLXNA3 Chemorepulsion A 1

(n)

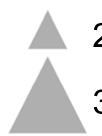

1

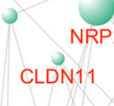

SPRYY1
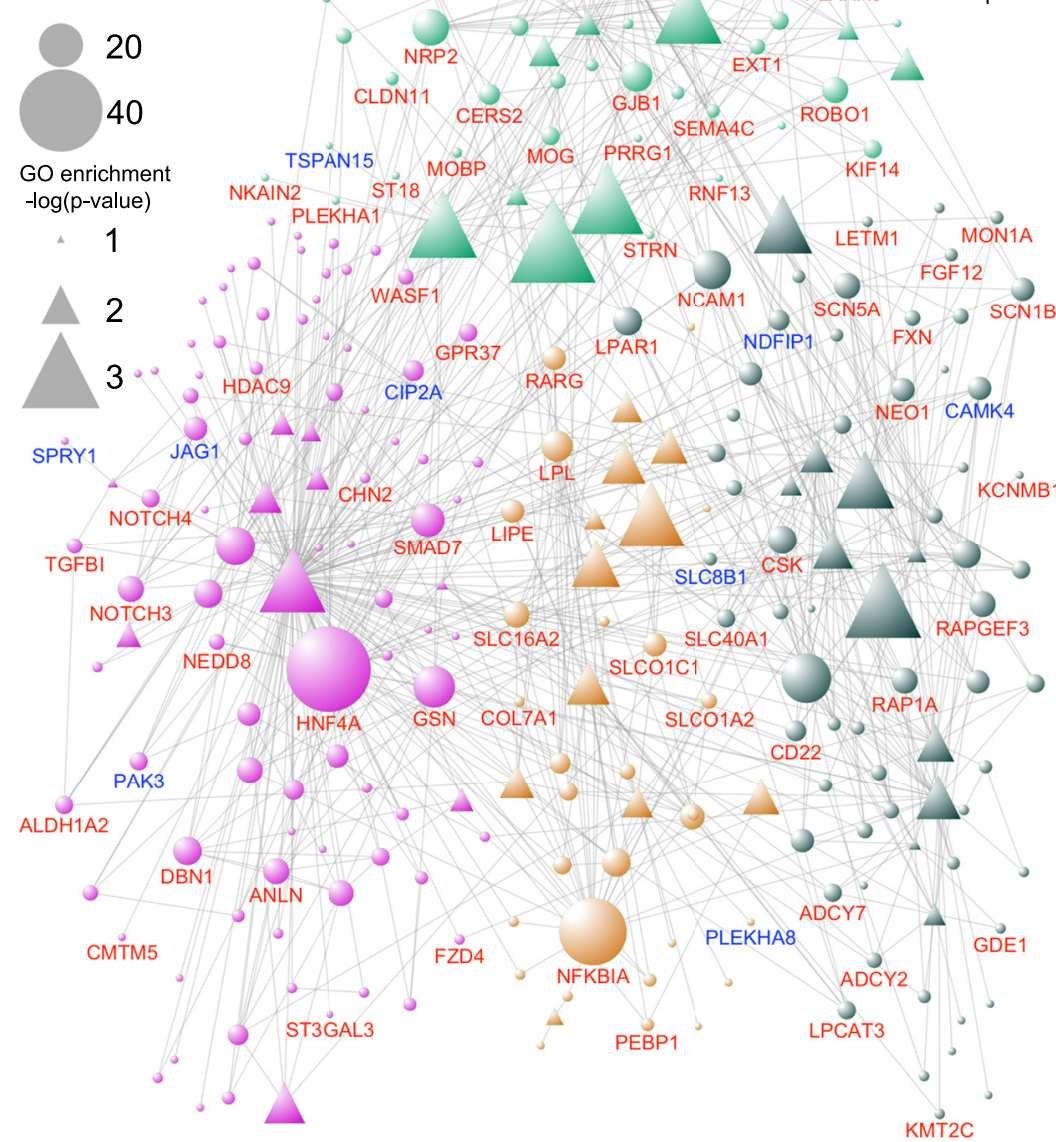

M2 - 35.4\%

Cell Movement

SMAD4 And TGF $\beta$ Signaling

Notch Signaling

CIP2A Signaling

M3 - $15.0 \%$

Uptake and Transport of T3

RXRA Signaling

Uptake, Transport, and

Storage of Lipid
M4 - 30.8\%

Iron and Metal Homeostasis Quantity of $\mathrm{Ca} 2+$

cAMP Mediated Signaling

Schizophrenia

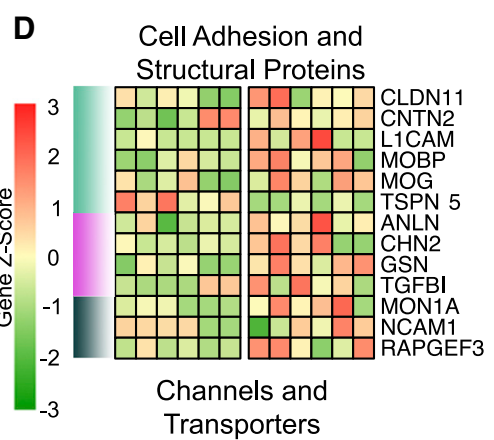

Transporters

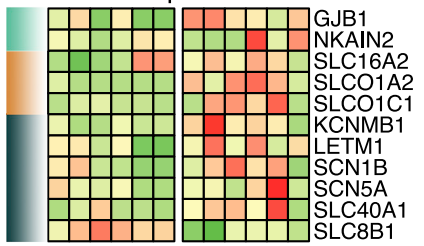

Enzymes

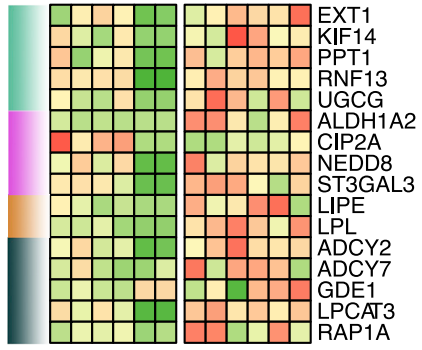

Kinases

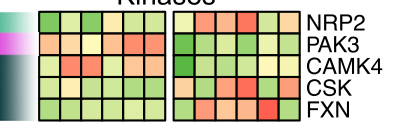

Ligands and

Secreted Proteins

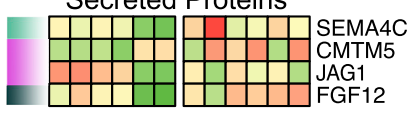

Receptors and

Downstream Components

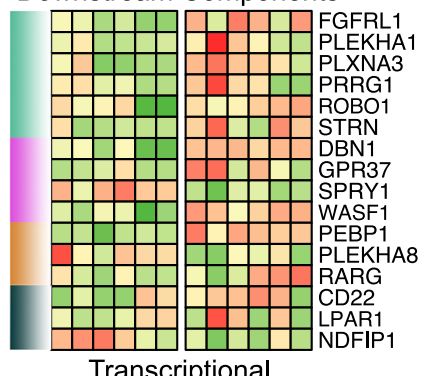

Regulators

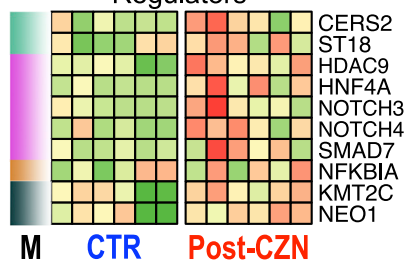

(legend on next page) 
in Figure 6; complete ontology listings and network table in Table S2). Community detection by modularity analysis was then carried out to aggregate closely related functions and genes (Bastian et al., 2009; Blondel et al., 2008). This analysis yielded four distinct modules (M1-M4), which individually identified distinct processes associated with the initiation of remyelination by cuprizone demyelination-mobilized hGPCs.

M1 revealed that the hGPCs recovering from cuprizone demyelination markedly upregulated their expression of myelinogenesis-associated genes, including MOG, MOBP, and CLDN11 (Goldman and Kuypers, 2015) (Figure 5C). Furthermore, several genes previously noted to be induced during oligodendrocyte differentiation and remyelination were also upregulated; these genes included ST18 (Najm et al., 2013), PLEKHA1 (Chen et al., 2015), and CMTM5 (Doyle et al., 2008), along with those shown to be necessary for appropriate maturation of oligodendrocytes: CERS2 (Imgrund et al., 2009), LPAR1 (García-Diaz et al., 2015), GSN (Zuchero et al., 2015), KIF14 (Fujikura et al., 2013), CNTN2 (Zoupi et al., 2018), ST3GAL3 (Yoo et al., 2015), and WASF1/WAVE1 (Kim et al., 2006). Interestingly, M1 further revealed that TCF7L2 signaling, a major driver of myelination (Hammond et al., 2015; Zhao et al., 2016), is also strongly upregulated in remyelinating hGPCs. Genes contributing to the migration and pathfinding of GPCs were also upregulated following cuprizone exposure; these genes included $R O B O 1$ (Liu et al., 2012) and the class 3 semaphorin co-receptors NRP2 (Boyd et al., 2013) and PLXNA3 (Xiang et al., 2012).

Within $\mathrm{M} 2$, a number of transcription factors and associated signal effectors vital to oligodendrocyte movement and differentiation were noted to be significantly enriched in the post-cuprizone hGPCs. These included SMAD4 (Choe et al., 2014), transforming growth factor $\beta$ (TGF- $\beta$ ) (McKinnon et al., 1993), and NOTCH (Park and Appel, 2003), as well as the pro-myelinogenic genes SMAD7 (Weng et al., 2012), PAK3 (Maglorius Renkilaraj et al., 2017), and NOTCH3 (Zaucker et al., 2013). In contrast, the Notch pathway inhibitor of differentiation JAG1 was sharply repressed (John et al., 2002), suggesting the incipient differentiation of these cells. Also localizing to this module and upregulated in remyelinating GPCs was GPR37, the expression of which attends and is necessary for oligodendrocyte differentiation (Smith et al., 2017; Yang et al., 2016). Interestingly, CIP2A, a transcriptional repressor of GPR37, was profoundly downregulated in cuprizone-mobilized hGPCs, again suggesting that cuprizone-demyelination triggers the active disinhibition of oligodendrocytic differentiation by previously quiescent parenchymal hGPCs (Yang et al., 2016).

M3 consisted of several functional categories strongly involved in myelination. These included transcripts involved in the transport and uptake of thyroid hormone and L-triiodothyronine (Almazan et al., 1985; Bhat et al., 1979). M3 also included genes associated with retinoid signaling, particularly RXRA and the retinoid receptor complex partner RARG, the upregulation of which was observed in hGPCs after cuprizone treatment (Huang et al., 2011; Tomaru et al., 2009). This is of particular significance, as this signaling family has previously been tied not only to developmental myelination (de la Fuente et al., 2015) but also to remyelination as well (Huang et al., 2011). M3 also included genes associated with cholesterol and lipid uptake, processes critical to myelination (Saher et al., 2005).

The fourth module included genes associated with the transport and homeostatic regulation of iron and other multivalent cations, which were upregulated following cuprizone demyelination. Iron, in particular, has been reported to be important in the regulation of oligodendrocytic differentiation and myelination (Connor and Fine, 1987; Morath and Mayer-Pröschel, 2001). In this regard, upregulation of MON1A and FXN, iron-metabolism-associated genes strongly dysregulated in multiple sclerosis lesions (Hametner et al., 2013), was observed in cuprizone-mobilized hGPCs. Similarly, we noted that genes encoding calcium regulatory proteins associated with myelin maturation, which included CASR, GSN, and TRPC3 (Cheli et al., 2015; Krasnow et al., 2018), were also increased in hGPCs after cuprizone demyelination, as were transcripts involved in cyclic AMP (cAMP) signaling, another modulator of oligodendrocyte differentiation, in part by crosstalk with GPR37 and GPR17 (Simon et al., 2016; Yang et al., 2016).

Overall, the pattern of differential gene expression by cuprizone-exposed hGPCs reflected in Figures 5 and 6 appears to define an expression network typifying that of early-progenitorderived remyelination. As such, these data indicate that when mobilized in response to antecedent cuprizone demyelination, hGPCs activated a coherent set of transcriptional programs that served to direct both oligodendrocytic differentiation and myelinogenesis. In addition, the many differentially expressed pathways operative in these hGPCs, relative to those that had not undergone previous cuprizone exposure and demyelination-induced mobilization, suggest that hGPCs resident within a chronically demyelinating environment acquire transcriptional patterns that may distinguish their functional competence and fate potential from that of otherwise naive resident glial progenitor cells.

\section{DISCUSSION}

The congenitally hypomyelinated shiverer mouse $\left(M B P^{\text {shi/shi }}\right)$ is a naturally occurring mutant that lacks MBP and thus cannot make

\footnotetext{
Figure 5. hGPC Transcriptional Networks Augur Compensatory Remyelination after Demyelination

Human glial chimeras were maintained on either a cuprizone (CZN)-containing or control diet from 12-24 weeks of age. Twelve weeks later, at 36 weeks, the mice were killed and their resident hGPCs isolated by CD140a-based FACS, which were then subjected to RNA-seq ( $n=6$ ).

(A) Principal-component analysis revealed tight clustering of hGPCs separated from post-CZN samples.

(B) Isolated hGPCs were enriched for genes indicative of an oligodendrocytic fate; gene expression representative of other lineages was minimal.

(C) A network was constructed from differentially expressed genes (circles) between post-CZN and CTR hGPCs (adjusted p < 0.05); significantly associated Gene Ontology (GO) annotations (triangles) identified those pertinent and functionally related genes (gene nodes) that were differentially active in CZN-mobilized hGPCs. Gene node size was determined by the degree of connectivity, and annotation node sizes scaled with their adjusted p values. Unsupervised modularity detection identified four modules (Ms) of closely related genes and annotations, for which a summary of annotations is provided with the percentage of total gene connectivity for each module. The complete list of differentially expressed genes is in Table S1, and complete network information is offered in Table S2. (D) A heatmap of genes identified in the previous GO network, organized by functional category and module (M) membership.
} 
A

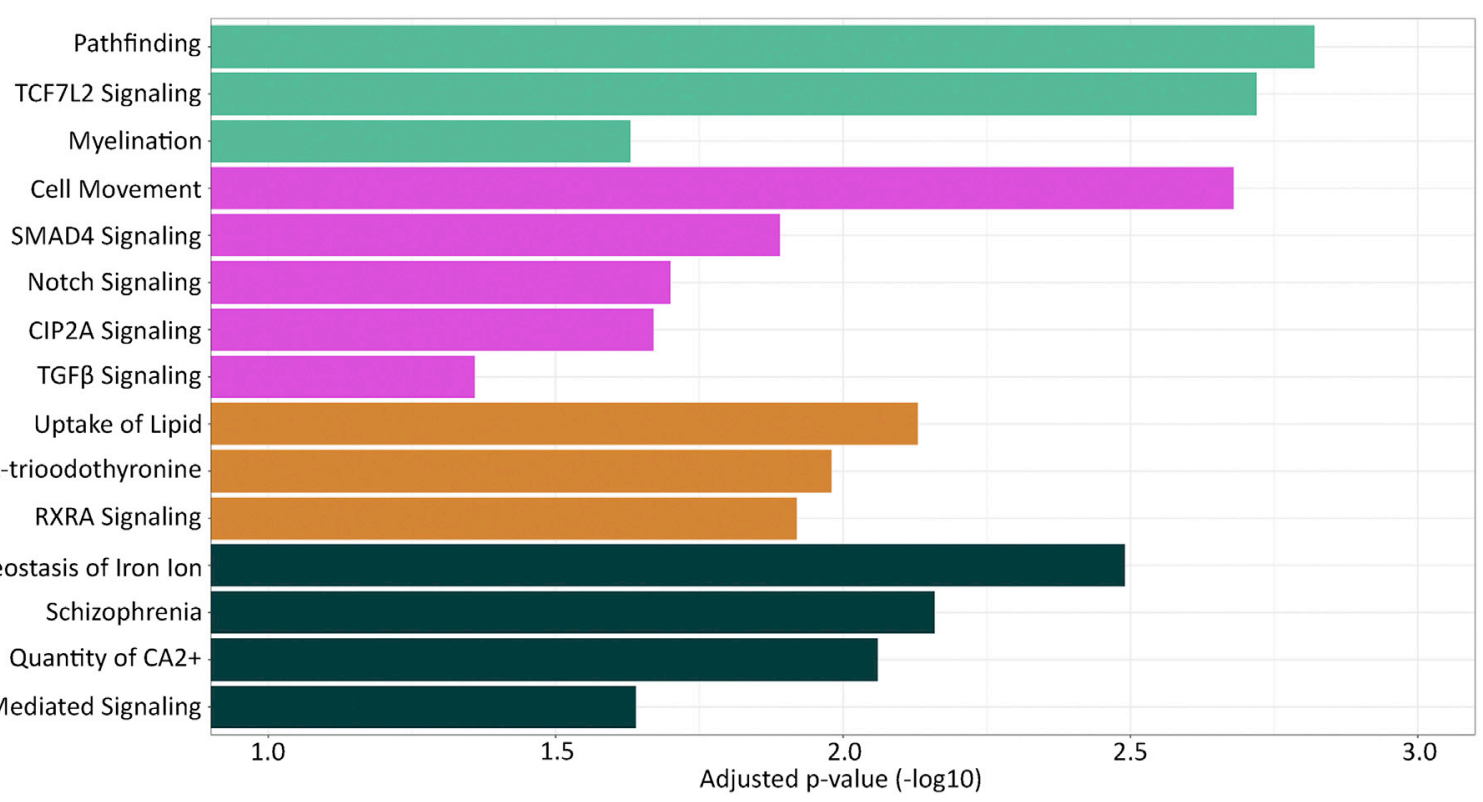

B

\begin{tabular}{|c|c|c|c|c|}
\hline Module & GO Annotation & Differentially Expressed Genes & $\begin{array}{c}-\log 10 \\
\text { (p-value) }\end{array}$ & $\begin{array}{l}\text { Activation } \\
\text { Z-Score }\end{array}$ \\
\hline \multirow{3}{*}{1} & Pathfinding & EXT1, NCAM1, NRP2, ROBO1 & 2.82 & NS \\
\hline & TCF7L2 Signaling & $\begin{array}{l}\text { ALDH1A2, ANLN, CASR, CERS2, CLDN11, GJB1, GSN, JAG1, MOBP, MOG, NKAIN2, PLEKHA1, } \\
\text { PRRG1, RAP1A, RNF13, SMAD7, ST18, STRN, TSPAN15 }\end{array}$ & 2.72 & 3.14 \\
\hline & Myelination & BCKDK, CERS2, DOCK7, GJB1, KIF14, L1CAM, MOBP, NCAM1, NFKBIA, RARG, SFTPA1, TG, UGCG & 1.63 & NS \\
\hline \multirow[t]{5}{*}{2} & Cell movement & $\begin{array}{l}\text { ADORA2B, AHCY, ANOS1, ARAP1, ATG16L1, BHLHE41, CAMK4, CAMSAP3, CARMIL1, CASR, } \\
\text { CCL16, CD22, CDCP1, CDH5, CELSR1, CFH, CHN2, CHRNA7, CIP2A, CLDN11, CLEC5A, CMTM5, } \\
\text { CNTN2, COL4A3, COL7A1, CORO1B, CSK, CYP2J2, DACT2, DBH, DBN1, DCN, DOCK5, DRD2, DTL, } \\
\text { EDIL3, EDN3, EFNA1, ELF3, F2RL3, FLT4, FOXP3, FURIN, FZD4, GIT1, GJB1, GPR37, GSN, HDAC9, } \\
\text { HNF4A, HOXB1, HRH4, IGSF8, IKZF1, IL16, IL4R, ITGAX, JAG1, KIF14, KIF1C, L1CAM, LGR6, LIPE, } \\
\text { LPAR1, LTB4R2, MAP2K6, MINK1, MIXL1, MOG, MYO18A, MYO1E, MYOCD, NCAM1, NCKAP1L, } \\
\text { NEO1, NFKBIA, NLRP3, NOTCH3, NOTCH4, NOX5, NR112, NRP2, ONECUT2, OPRM1, P4HA2, PAK3, } \\
\text { PARVG, PAX5, PCSK6, PEBP1, PLD1, PLXNA3, PRUNE1, PTPRH, RALBP1, RAP1A, RAPGEF3, RARG, } \\
\text { ROBO1, RUVBL2, SCN1B, SCN5A, SEMA4C, SFTPA1, SH3BP1, SIGLEC8, SMAD7, SP100, SPN, } \\
\text { ST3GAL3, TARS, TFAP4, TG, TGFBI, TMF1, TNFRSF11A, TNFRSF25, TRPM8, TSC22D3, VPS18, } \\
\text { WASF1, XCR1, ZNF580 }\end{array}$ & 2.68 & 3.05 \\
\hline & SMAD4 Signaling & CAB39, CDH5, CYP17A1, JAG1, HNF4A, LPAR1, NFKBIA, NOTCH3, PAK3, SMAD7 & 1.89 & NS \\
\hline & Notch Signaling & FURIN, JAG1, NOTCH3, NOTCH4 & 1.70 & 1.00 \\
\hline & CIP2A Signaling & CIP2A, DCN, GPR37, SLC22A18, TUBA4A & 1.67 & 2.24 \\
\hline & TGF $\beta$ Signaling & FOXP3, IL16, JAG1, SPRY1, TGFBI & 1.36 & NS \\
\hline \multirow{3}{*}{3} & Uptake of lipid & AKR1C1, KMT2C, LIPE, LPCAT3, LPL, NR112, PEBP1, SFTPA1, SLCO1A2, SLCO1C1, STRA6 & 2.13 & 2.17 \\
\hline & \begin{tabular}{|l|} 
Uptake of L- \\
triiodothyronine
\end{tabular} & SLC16A2, SLCO1C1 & 1.98 & NS \\
\hline & RXRA Signaling & AHCY, AKR1C1, ALDH1A2, HNF4A, HOXB1, LPL, RARG, SLC16A2, STRA6 & 1.92 & 1.27 \\
\hline \multirow{4}{*}{4} & $\begin{array}{l}\text { Homeostasis of Iron } \\
\text { Ion }\end{array}$ & ANK1, FXN, MON1A, NDFIP1, NEO1, SLC4OA1 & 2.49 & NS \\
\hline & Schizophrenia & $\begin{array}{l}\text { ADCY7, CHRNA2, CHRNA7, CLDN11, DAAM2, DRD2, GABRR2, GPR37, GSN, GTF2IRD1, KMT2C, } \\
\text { NBPF8, NCAM1, NEDD8, NFKBIA, NOTCH4, OPRM1, PCLO, PCSK1, PITPNM1, RARG, RBFOX1, } \\
\text { RIMS3, ROBO1, SCN1B, SCN4A, SCN5A, STRA6 }\end{array}$ & 2.16 & NS \\
\hline & Quantity of $\mathrm{Ca} 2+$ & $\begin{array}{l}\text { AVPR1A, CASR, CD22, CHRNA7, CSK, DCN, DRD2, EDN3, F2RL3, GSN, HNF4A, HRH4, L1CAM, } \\
\text { LAIR1, LTB4R2, NCAM1, OPRM1, OR51E2, RAPGEF3, SFTPA1, SPN, TNFRSF11A, TRIM21, TRPC3, } \\
\text { TRPM8, WFS1 }\end{array}$ & 2.06 & 2.94 \\
\hline & $\begin{array}{l}\text { cAMP-Mediated } \\
\text { Signaling }\end{array}$ & ADCY2, ADCY7, ADORA2B, CAMK4, DRD2, GDE1, LPAR1, OPRM1, PKIG, RAP1A, RAPGEF3, XCR1 & 1.64 & 2.31 \\
\hline
\end{tabular}


compact myelin. We had previously found that the intracerebral injection of hGPCs into neonatal shiverer mice results in the widespread dispersal of the human donor cells, followed by their oligodendrocytic differentiation and myelinogenesis (Windrem et al., 2004, 2008). This ultimately leads to the complete or near-complete myelination of the recipient's brain, brainstem, and spinal cord, attended by the clinical rescue of a large proportion of the transplanted mice. Yet, notwithstanding the robust developmental myelination of the host CNS by neonatally delivered hGPCs, in order for hGPC delivery to be a viable regenerative strategy for treating adult demyelination-especially as occurs in multicentric and diffuse myelin loss - the donor cells must be capable of migrating within and myelinating adult brain parenchyma.

In adults, oligodendrocytic loss contributes to diseases as diverse as hypertensive and diabetic white matter loss, traumatic spinal cord and brain injury, multiple sclerosis and its variants, and even the age-related white matter loss of the subcortical dementias. All of these conditions are potential targets of glial progenitor cell replacement therapy, recognizing that the adult disease environment may limit this approach in a disease-specific fashion (Goldman, 2017; Goldman et al., 2012). For instance, the chronically ischemic brain tissue of diabetics with small vessel disease may require aggressive treatment of the underlying vascular insufficiency before any cell replacement strategy may be considered. Similarly, the inflammatory disease environments of multiple sclerosis as well as of many of the leukodystrophies present their own challenges, which need to be overcome before cell-based remyelination can succeed (Franklin and Ffrench-Constant, 2008, 2017; Goldman, 2016; Ip et al., 2006); these include participation by mobilized GPCs in the inflammatory response, potentially complicating therapeutic efforts further (Falcão et al., 2018). Nonetheless, current diseasemodifying strategies for treating both vascular and autoimmune diseases have advanced to the point where stabilization of the disease environment can often be accomplished, such that transplant-based remyelination for the structural repair of demyelinated adult white matter may now be feasible.

Despite concerns as to the ability of glial progenitor cells to remyelinate axons in disease environments, such as those associated with multiple sclerosis and the periventricular leukomalacia of cerebral palsy, a number of studies have pointed to the cellintrinsic nature of oligodendrocytic differentiation block in these cases (Fancy et al., 2009, 2014). These studies have suggested that the inability of parenchymal glial progenitors to produce myelinating oligodendrocytes in these conditions is a function of stable epigenetic blocks in the differentiation potential of these cells, imparted by the specific disease process or its antecedents. Newly introduced naive hGPCs might, thus, be expected to exercise unfettered differentiation and myelination competence in host brains and, as such, be able to remyelinate previ- ously demyelinated axons. Indeed, several prior studies have indicated the ability of transplanted oligodendrocyte progenitors to remyelinate adult-demyelinated central axons (Duncan et al., 2009; Mozafari et al., 2015). To define the competence of hGPCs to remyelinate axons when delivered to the demyelinated adult brain, we used two different antigenic phenotypes of GPCs, respectively defined as $\mathrm{CD}_{140 \mathrm{a}^{+}}$and $\mathrm{A} 2 \mathrm{~B} 5^{+} / \mathrm{PSA}-\mathrm{NCAM}^{-}$, each derived from fetal human brain tissue (Sim et al., 2011; Windrem et al., 2008). These antigenic phenotypes are largely but not completely homologous; the CD140a phenotype is the major fraction of, and largely subsumed within, the $\mathrm{A} 2 \mathrm{~B}^{+} \%$ PSA-NCAM $^{-}$pool (Sim et al., 2011). We assessed the dispersal and myelination competence of these cell types in two distinct adult models of myelin deficiency, namely, the congenitally hypomyelinated shiverer mouse, as well as the normally myelinated adult mouse, and the cuprizone-treated demyelinated adult mouse.

In each of these model systems, the transplanted hGPCs effectively restored myelin to the host brain. Donor-derived myelination was robust when cells were delivered to the adult shiverer brain, just as we had previously reported after hGPC transplants in neonatal shiverer mice. The design of this experiment was intended to mimic what might be encountered in the post-natal treatment of a hypomyelinating leukodystrophy, and the effective progenitor cell dispersal and myelination that we observed augurs well for the potential use of this approach in the treatment of children with congenital leukodystrophies. Similarly, donor hGPC-derived oligodendrocyte differentiation and axonal remyelination proved robust in response to cuprizone demyelination, whether by hGPCs already resident within the adult demyelinated brains or by those transplanted during and after demyelination. This latter set of experiments, in particular, provided an important proof-of-principle, showing that hGPCs could remyelinate axons that had already been myelinated in the past and that were then demyelinated in the setting of oligodendrocyte loss; precisely such a scenario might be anticipated in disorders such as progressive multiple sclerosis, in which the remyelination of stably denuded residual axons might be expected to confer a functional benefit.

Indeed, in each of these experimental paradigms, we found that the hGPCs, whether engrafted neonatally or transplanted into adults, effectively dispersed throughout the forebrains, even in normally myelinated mice, and differentiated as oligodendroglia and myelinated demyelinated lesions as these evolved or were encountered. These data suggest that transplanted fetal hGPCs are competent to disperse broadly and differentiate as myelinogenic cells in the adult brain and, critically, that they are able to remyelinate previously myelinated axons that have experienced myelin loss.

Having established the potential of hGPCs as myelinogenic vectors, we then used RNA-seq of hGPCs extracted from the

Figure 6. Enrichment of Remyelination-Associated Pathways in Cuprizone-Exposed hGPCs

(A) The significantly enriched functional categories highlighted in Figure 5C are organized here by color-defined modules. Enrichment was determined by Fisher's exact test in Ingenuity Pathway Analysis.

(B) Genes differentially expressed by CZN-exposed hGPCs relative to controls, which contributed to these differentially enriched pathways. Genes upregulated after cuprizone exposure are in red and those downregulated in blue. Activation $Z$ scores are also provided for those pathways for which collective gene expression implies activation (red) or inhibition (blue), following CZN exposure in post-CZN versus CTR hGPCs. Activation $Z$ scores $>1$ were deemed significant. 
brains in which they had been resident during cuprizone exposure to assess the transcriptional response of these cells to demyelination and initial remyelination. By this means, we correlated the demyelination-associated mobilization of resident hGPCs to their subsequent transcriptional patterns, so as to identify - in human cells isolated directly from the in vivo environment-those genes and pathways whose targeting might permit the therapeutic modulation of both progenitor recruitment and differentiated fate.

We found that mobilized hGPCs expressed a transcriptional signature consistent with early remyelination, as has been noted with demyelination-mobilized murine progenitors as well (Moyon et al., 2015). Yet, human and mouse glial progenitors are quite different phenotypes, and are distinct in their transcriptional signatures (Lovatt et al., 2007; Sim et al., 2006, 2009; Zhang et al., 2016), lineage restriction (Nunes et al., 2003), and daughter cell morphologies (Oberheim et al., 2009). Accordingly, we noted a number of activated pathways in demyelination-triggered hGPCs not previously noted in models of murine demyelination. These comprised a number of upstream directors of oligodendroglial fate choice and differentiation, which included TCF7L2, TGF- $\beta / S M A D 4$, and NOTCH-driven pathways. The activation of each of these pathways was linked to the demyelination-associated disinhibition of differentiation by these parenchymal hGPCs, the oligodendrocytic maturation of which then enabled the compensatory remyelination of denuded axons. In addition, besides the activation of differentiation-associated pathways in demyelination-stimulated hGPCs, our data also suggested the critical importance during early remyelination of pathways enabling iron transport and metabolism, as well as those facilitating cholesterol and lipid uptake, all of which are critically important to oligodendrocytic myelinogenesis. Interestingly, the identification of these pathways as differentially upregulated during remyelination suggests that the efficiencies of both myelinogenesis and myelin maturation might be further potentiated by metabolic optimization, and potentially even by dietary modulation. Together, these studies establish an operational rationale for assessing the ability of hGPCs to remyelinate demyelinated lesions of the adult human brain, while providing a promising set of molecular targets for the modulation of this process in human cells.

\section{STAR $\star$ METHODS}

Detailed methods are provided in the online version of this paper and include the following:

- KEY RESOURCES TABLE

- RESOURCE AVAILABILITY

- Lead Contact

- Materials availability

Data and Code Availability

- EXPERIMENTAL MODEL AND SUBJECT DETAILS

O Animal models and transplantation

O Cells

- METHOD DETAILS

○ Quantitative histology

O Functional assessment
- QUANTIFICATION AND STATISTICAL ANALYSIS

O Statistics

O RNA-Sequencing and analysis

\section{SUPPLEMENTAL INFORMATION}

Supplemental Information can be found online at https://doi.org/10.1016/j. celrep.2020.107658.

\section{ACKNOWLEDGMENTS}

This work was support by NINDS grants R01NS110776 and R01NS75345, the Dr. Miriam and Sheldon G. Adelson Medical Research Foundation, the G. Harold and Leila Y. Mathers Charitable Foundation, the NY Stem Cell Research Program (NYSTEM), the Oscine Corporation, Sana Biotechnology, the Novo Nordisk Foundation, and the Lundbeck Foundation. We are grateful to Karen Bentley and Chad Galloway of the URMC Shared Resource Laboratory for assistance with electron microscopy, to Dr. Abdellatif Benraiss for designing the graphic abstract, and to Dr. Thomas G. Hampton of MouseSpecifics, Inc., for his assistance with our functional motor analyses.

\section{AUTHOR CONTRIBUTIONS}

D.C.-M., S.S., and N.J.K. prepared the GPCs used in the study, including the cytometry and sorting; S.S. and L.Z. performed the transplants; L.Z. and S.S. did the histological processing, imaging, and quantitative analyses; Y.L. and M.N. conducted the transcallosal conduction velocity assessments; J.N.M. performed the genomic analyses; M.W. and S.A.G. designed the study; and S.A.G. analyzed all data and wrote the manuscript.

\section{DECLARATION OF INTERESTS}

S.A.G. is a co-founder of the Oscine Corporation and receives sponsored research support and consulting income from Oscine. S.A.G. and M.W. are co-inventors on patents covering the therapeutic uses of human glial progenitors, which have been licensed by the University of Rochester to Oscine. None of the other authors have any known conflicts of interest in regard to this work.

Received: July 11, 2019

Revised: February 14, 2020

Accepted: April 18, 2020

Published: May 19, 2020

\section{REFERENCES}

Almazan, G., Honegger, P., and Matthieu, J.M. (1985). Triiodothyronine stimulation of oligodendroglial differentiation and myelination. A developmental study. Dev. Neurosci. 7, 45-54.

Archer, D.R., Levén, S., and Duncan, I.D. (1994). Myelination by cryopreserved xenografts and allografts in the myelin-deficient rat. Exp. Neurol. 125, 268-277.

Archer, D.R., Cuddon, P.A., Lipsitz, D., and Duncan, I.D. (1997). Myelination of the canine central nervous system by glial cell transplantation: a model for repair of human myelin disease. Nat. Med. 3, 54-59.

Bastian, M., Heymann, S., and Jacomy, M. (2009). Gephi: An Open Source Software for Exploring and Manipulating Networks.https://gephi.org/ publications/gephi-bastian-feb09.pdf.

Bhat, N.R., Sarlieve, L.L., Rao, G.S., and Pieringer, R.A. (1979). Investigations on myelination in vitro. Regulation by thyroid hormone in cultures of dissociated brain cells from embryonic mice. J. Biol. Chem. 254, 9342-9344.

Blondel, V.D., Guillaume, J.-L., Lambiotte, R., and Lefebvre, E. (2008). Fast unfolding of communities in large networks. J. Stat. Mech. 10, 10008.

Bolger, A.M., Lohse, M., and Usadel, B. (2014). Trimmomatic: a flexible trimmer for Illumina sequence data. Bioinformatics 30, 2114-2120. 


\section{CellPress}

Boyd, A., Zhang, H., and Williams, A. (2013). Insufficient OPC migration into demyelinated lesions is a cause of poor remyelination in MS and mouse models. Acta Neuropathol. 125, 841-859.

Brüstle, O., Jones, K.N., Learish, R.D., Karram, K., Choudhary, K., Wiestler, O.D., Duncan, I.D., and McKay, R.D. (1999). Embryonic stem cell-derived glial precursors: a source of myelinating transplants. Science 285, 754-756.

Buchet, D., Garcia, C., Deboux, C., Nait-Oumesmar, B., and Baron-Van Evercooren, A. (2011). Human neural progenitors from different foetal forebrain regions remyelinate the adult mouse spinal cord. Brain 134, 1168-1183.

Cheli, V.T., Santiago González, D.A., Spreuer, V., and Paez, P.M. (2015). Voltage-gated $\mathrm{Ca} 2+$ entry promotes oligodendrocyte progenitor cell maturation and myelination in vitro. Exp. Neurol. 265, 69-83.

Chen, Y., Mei, R., Teng, P., Yang, A., Hu, X., Zhang, Z., Qiu, M., and Zhao, X. (2015). TAPP1 inhibits the differentiation of oligodendrocyte precursor cells via suppressing the Mek/Erk pathway. Neurosci. Bull. 31, 517-526.

Choe, Y., Huynh, T., and Pleasure, S.J. (2014). Migration of oligodendrocyte progenitor cells is controlled by transforming growth factor $\beta$ family proteins during corticogenesis. J. Neurosci. 34, 14973-14983.

Connor, J.R., and Fine, R.E. (1987). Development of transferrin-positive oligodendrocytes in the rat central nervous system. J. Neurosci. Res. 17, 51-59.

Connor, J.R., Roskams, A.J., Menzies, S.L., and Williams, M.E. (1993). Transferrin in the central nervous system of the shiverer mouse myelin mutant. J. Neurosci. Res. 36, 501-507.

de la Fuente, A.G., Errea, O., van Wijngaarden, P., Gonzalez, G.A., Kerninon, C., Jarjour, A.A., Lewis, H.J., Jones, C.A., Nait-Oumesmar, B., Zhao, C., et al. (2015). Vitamin D receptor-retinoid $X$ receptor heterodimer signaling regulates oligodendrocyte progenitor cell differentiation. J. Cell Biol. 211, 975-985.

Dobin, A., Davis, C.A., Schlesinger, F., Drenkow, J., Zaleski, C., Jha, S., Batut, P., Chaisson, M., and Gingeras, T.R. (2013). STAR: ultrafast universal RNA-seq aligner. Bioinformatics 29, 15-21.

Doyle, J.P., Dougherty, J.D., Heiman, M., Schmidt, E.F., Stevens, T.R., Ma, G., Bupp, S., Shrestha, P., Shah, R.D., Doughty, M.L., et al. (2008). Application of a translational profiling approach for the comparative analysis of CNS cell types. Cell 135, 749-762.

Duncan, I.D., Brower, A., Kondo, Y., Curlee, J.F., Jr., and Schultz, R.D. (2009). Extensive remyelination of the CNS leads to functional recovery. Proc. Natl. Acad. Sci. USA 106, 6832-6836.

Ehrlich, M., Mozafari, S., Glatza, M., Starost, L., Velychko, S., Hallmann, A.L., Cui, Q.L., Schambach, A., Kim, K.P., Bachelin, C., et al. (2017). Rapid and efficient generation of oligodendrocytes from human induced pluripotent stem cells using transcription factors. Proc. Natl. Acad. Sci. USA 114, E2243E2252.

Falcão, A.M., van Bruggen, D., Marques, S., Meijer, M., Jäkel, S., Agirre, E., Samudyata, Floriddia, E.M., Vanichkina, D.P., Ffrench-Constant, C., et al. (2018). Disease-specific oligodendrocyte lineage cells arise in multiple sclerosis. Nat. Med. 24, 1837-1844.

Fancy, S.P., Baranzini, S.E., Zhao, C., Yuk, D.I., Irvine, K.A., Kaing, S., Sanai, N., Franklin, R.J., and Rowitch, D.H. (2009). Dysregulation of the Wnt pathway inhibits timely myelination and remyelination in the mammalian CNS. Genes Dev. 23, 1571-1585.

Fancy, S.P., Harrington, E.P., Baranzini, S.E., Silbereis, J.C., Shiow, L.R., Yuen, T.J., Huang, E.J., Lomvardas, S., and Rowitch, D.H. (2014). Parallel states of pathological Wnt signaling in neonatal brain injury and colon cancer. Nat. Neurosci. 17, 506-512.

Ferrari Bardile, C., Garcia-Miralles, M., Caron, N.S., Rayan, N.A., Langley, S.R., Harmston, N., Rondelli, A.M., Teo, R.T.Y., Waltl, S., Anderson, L.M., et al. (2019). Intrinsic mutant HTT-mediated defects in oligodendroglia cause myelination deficits and behavioral abnormalities in Huntington disease. Proc. Natl. Acad. Sci. USA 116, 9622-9627.

Franklin, R.J.M., and Ffrench-Constant, C. (2008). Remyelination in the CNS: from biology to therapy. Nat. Rev. Neurosci. 9, 839-855.
Franklin, R.J.M., and Ffrench-Constant, C. (2017). Regenerating CNS myelin from mechanisms to experimental medicines. Nat. Rev. Neurosci. 18, 753-769.

Franklin, R.J., and Goldman, S.A. (2015). Glia Disease and Repair-Remyelination. Cold Spring Harb. Perspect. Biol. 7, a020594.

Fujikura, K., Setsu, T., Tanigaki, K., Abe, T., Kiyonari, H., Terashima, T., and Sakisaka, T. (2013). Kif14 mutation causes severe brain malformation and hypomyelination. PLoS One 8, e53490.

García-Díaz, B., Riquelme, R., Varela-Nieto, I., Jiménez, A.J., de Diego, I., Gómez-Conde, A.l., Matas-Rico, E., Aguirre, J.A., Chun, J., Pedraza, C., et al. (2015). Loss of lysophosphatidic acid receptor LPA1 alters oligodendrocyte differentiation and myelination in the mouse cerebral cortex. Brain Struct. Funct. 220, 3701-3720.

Goldman, S.A. (2016). Stem and progenitor cell-based therapy of the central nervous system: Hopes, hype, and wishful thinking. Cell Stem Cell 18, $174-188$

Goldman, S.A. (2017). Progenitor cell-based treatment of glial disease. Prog. Brain Res. 231, 165-189.

Goldman, S.A., and Kuypers, N.J. (2015). How to make an oligodendrocyte. Development 142, 3983-3995.

Goldman, S.A., Nedergaard, M., and Windrem, M.S. (2012). Glial progenitor cell-based treatment and modeling of neurological disease. Science 338 , 491-495.

Hametner, S., Wimmer, I., Haider, L., Pfeifenbring, S., Brück, W., and Lassmann, H. (2013). Iron and neurodegeneration in the multiple sclerosis brain. Ann. Neurol. 74, 848-861.

Hammond, E., Lang, J., Maeda, Y., Pleasure, D., Angus-Hill, M., Xu, J., Horiuchi, M., Deng, W., and Guo, F. (2015). The Wnt effector transcription factor 7-like 2 positively regulates oligodendrocyte differentiation in a manner independent of Wnt/ $\beta$-catenin signaling. J. Neurosci. 35, 5007-5022.

Hibbits, N., Pannu, R., Wu, T.J., and Armstrong, R.C. (2009). Cuprizone demyelination of the corpus callosum in mice correlates with altered social interaction and impaired bilateral sensorimotor coordination. ASN Neuro. 1, e00013. Hibbits, N., Yoshino, J., Le, T.Q., and Armstrong, R.C. (2012). Astrogliosis during acute and chronic cuprizone demyelination and implications for remyelination. ASN Neuro 4, 393-408.

Huang, J.K., Jarjour, A.A., Nait Oumesmar, B., Kerninon, C., Williams, A., Krezel, W., Kagechika, H., Bauer, J., Zhao, C., Baron-Van Evercooren, A., et al. (2011). Retinoid X receptor gamma signaling accelerates CNS remyelination. Nat. Neurosci. 14, 45-53.

Imgrund, S., Hartmann, D., Farwanah, H., Eckhardt, M., Sandhoff, R., Degen, J., Gieselmann, V., Sandhoff, K., and Willecke, K. (2009). Adult ceramide synthase 2 (CERS2)-deficient mice exhibit myelin sheath defects, cerebellar degeneration, and hepatocarcinomas. J. Biol. Chem. 284, 33549-33560.

Ip, C.W., Kroner, A., Bendszus, M., Leder, C., Kobsar, I., Fischer, S., Wiendl, H., Nave, K.A., and Martini, R. (2006). Immune cells contribute to myelin degeneration and axonopathic changes in mice overexpressing proteolipid protein in oligodendrocytes. J. Neurosci. 26, 8206-8216.

John, G.R., Shankar, S.L., Shafit-Zagardo, B., Massimi, A., Lee, S.C., Raine, C.S., and Brosnan, C.F. (2002). Multiple sclerosis: re-expression of a developmental pathway that restricts oligodendrocyte maturation. Nat. Med. 8, 11151121.

Kim, H.-J., DiBernardo, A.B., Sloane, J.A., Rasband, M.N., Solomon, D., Kosaras, B., Kwak, S.P., and Vartanian, T.K. (2006). WAVE1 is required for oligodendrocyte morphogenesis and normal CNS myelination. J. Neurosci. 26, 58495859.

Kondo, Y., and Duncan, I.D. (2016). Myelin repair by transplantation of myelinforming cells in globoid cell leukodystrophy. J. Neurosci. Res. 94, 1195-1202. Krasnow, A.M., Ford, M.C., Valdivia, L.E., Wilson, S.W., and Attwell, D. (2018). Regulation of developing myelin sheath elongation by oligodendrocyte calcium transients in vivo. Nat. Neurosci. 21, 24-28.

Lee, Y., Morrison, B.M., Li, Y., Lengacher, S., Farah, M.H., Hoffman, P.N., Liu, Y., Tsingalia, A., Jin, L., Zhang, P.W., et al. (2012). Oligodendroglia 
metabolically support axons and contribute to neurodegeneration. Nature 487, 443-448.

Li, B., and Dewey, C.N. (2011). RSEM: accurate transcript quantification from RNA-Seq data with or without a reference genome. BMC Bioinformatics 12, 323.

Liu, X., Lu, Y., Zhang, Y., Li, Y., Zhou, J., Yuan, Y., Gao, X., Su, Z., and He, C. (2012). Slit2 regulates the dispersal of oligodendrocyte precursor cells via Fyn/ RhoA signaling. J. Biol. Chem. 287, 17503-17516.

Lovatt, D., Sonnewald, U., Waagepetersen, H.S., Schousboe, A., He, W., Lin, J.H., Han, X., Takano, T., Wang, S., Sim, F.J., et al. (2007). The transcriptome and metabolic gene signature of protoplasmic astrocytes in the adult murine cortex. J. Neurosci. 27, 12255-12266.

Love, M.I., Huber, W., and Anders, S. (2014). Moderated estimation of fold change and dispersion for RNA-seq data with DESeq2. Genome Biol. 15, 550.

Maglorius Renkilaraj, M.R.L., Baudouin, L., Wells, C.M., Doulazmi, M., Wehrlé, R., Cannaya, V., Bachelin, C., Barnier, J.V., Jia, Z., Nait Oumesmar, B., et al. (2017). The intellectual disability protein PAK3 regulates oligodendrocyte precursor cell differentiation. Neurobiol. Dis. 98, 137-148.

Matsushima, G.K., and Morell, P. (2001). The neurotoxicant, cuprizone, as a model to study demyelination and remyelination in the central nervous system. Brain Pathol. 11, 107-116.

McKinnon, R.D., Piras, G., Ida, J.A., Jr., and Dubois-Dalcq, M. (1993). A role for TGF-beta in oligodendrocyte differentiation. J. Cell Biol. 121, 1397-1407.

Morath, D.J., and Mayer-Pröschel, M. (2001). Iron modulates the differentiation of a distinct population of glial precursor cells into oligodendrocytes. Dev. Biol. 237, 232-243.

Morell, P., Barrett, C.V., Mason, J.L., Toews, A.D., Hostettler, J.D., Knapp, G.W., and Matsushima, G.K. (1998). Gene expression in brain during cuprizone-induced demyelination and remyelination. Mol. Cell. Neurosci. 12, 220-227.

Moyon, S., Dubessy, A.L., Aigrot, M.S., Trotter, M., Huang, J.K., Dauphinot, L., Potier, M.C., Kerninon, C., Melik Parsadaniantz, S., Franklin, R.J., and Lubetzki, C. (2015). Demyelination causes adult CNS progenitors to revert to an immature state and express immune cues that support their migration. J. Neurosci. 35, 4-20.

Mozafari, S., Laterza, C., Roussel, D., Bachelin, C., Marteyn, A., Deboux, C., Martino, G., and Baron-Van Evercooren, A. (2015). Skin-derived neural precursors competitively generate functional myelin in adult demyelinated mice. J. Clin. Invest. 125, 3642-3656.

Najm, F.J., Lager, A.M., Zaremba, A., Wyatt, K., Caprariello, A.V., Factor, D.C., Karl, R.T., Maeda, T., Miller, R.H., and Tesar, P.J. (2013). Transcription factormediated reprogramming of fibroblasts to expandable, myelinogenic oligodendrocyte progenitor cells. Nat. Biotechnol. 31, 426-433.

Nicaise, A.M., Banda, E., Guzzo, R.M., Russomanno, K., Castro-Borrero, W., Willis, C.M., Johnson, K.M., Lo, A.C., and Crocker, S.J. (2017). iPS-derived neural progenitor cells from PPMS patients reveal defect in myelin injury response. Exp. Neurol. 288, 114-121.

Nicaise, A.M., Wagstaff, L.J., Willis, C.M., Paisie, C., Chandok, H., Robson, P., Fossati, V., Williams, A., and Crocker, S.J. (2019). Cellular senescence in progenitor cells contributes to diminished remyelination potential in progressive multiple sclerosis. Proc. Natl. Acad. Sci. USA 116, 9030-9039.

Nishiyama, A., Komitova, M., Suzuki, R., and Zhu, X. (2009). Polydendrocytes (NG2 cells): multifunctional cells with lineage plasticity. Nat. Rev. Neurosci. 10, 9-22.

Nunes, M.C., Roy, N.S., Keyoung, H.M., Goodman, R.R., McKhann, G., 2nd, Jiang, L., Kang, J., Nedergaard, M., and Goldman, S.A. (2003). Identification and isolation of multipotential neural progenitor cells from the subcortical white matter of the adult human brain. Nat. Med. 9, 439-447.

Oberheim, N.A., Takano, T., Han, X., He, W., Lin, J.H., Wang, F., Xu, Q., Wyatt, J.D., Pilcher, W., Ojemann, J.G., et al. (2009). Uniquely hominid features of adult human astrocytes. J. Neurosci. 29, 3276-3287.

Osipovitch, M., Asenjo Martinez, A., Mariani, J.N., Cornwell, A., Dhaliwal, S., Zou, L., Chandler-Militello, D., Wang, S., Li, X., Benraiss, S.J., et al. (2019). Hu- man ESC-Derived Chimeric Mouse Models of Huntington's Disease Reveal Cell-Intrinsic Defects in Glial Progenitor Cell Differentiation. Cell Stem Cell 24, 107-122.e107.

Park, H.C., and Appel, B. (2003). Delta-Notch signaling regulates oligodendrocyte specification. Development 130, 3747-3755.

Piao, J., Major, T., Auyeung, G., Policarpio, E., Menon, J., Droms, L., Gutin, P., Uryu, K., Tchieu, J., Soulet, D., and Tabar, V. (2015). Human embryonic stem cell-derived oligodendrocyte progenitors remyelinate the brain and rescue behavioral deficits following radiation. Cell Stem Cell 16, 198-210.

R Core Team (2017). R: A Language and environment for statistical computing (R Foundation for Statistical Computing).

Risso, D., Schwartz, K., Sherlock, G., and Dudoit, S. (2011). GC-content normalization for RNA-Seq data. BMC Bioinformatics 12, 480.

Risso, D., Ngai, J., Speed, T.P., and Dudoit, S. (2014). Normalization of RNAseq data using factor analysis of control genes or samples. Nat. Biotechnol. 32, 896-902.

Roy, N., Windrem, M., and Goldman, S.A. (2004). Progenitor cells of the adult white matter. In Myelin Biology and Disorders, R. Lazzarini, ed. (Elsevier), pp. 259-287.

Saher, G., Brügger, B., Lappe-Siefke, C., Möbius, W., Tozawa, R., Wehr, M.C., Wieland, F., Ishibashi, S., and Nave, K.-A. (2005). High cholesterol level is essential for myelin membrane growth. Nat. Neurosci. 8, 468-475.

Shannon, P., Markiel, A., Ozier, O., Baliga, N.S., Wang, J.T., Ramage, D., Amin, N., Schwikowski, B., and Ideker, T. (2003). Cytoscape: a software environment for integrated models of biomolecular interaction networks. Genome Res. 13, 2498-2504.

Shihabuddin, L.S., Numan, S., Huff, M.R., Dodge, J.C., Clarke, J., Macauley, S.L., Yang, W., Taksir, T.V., Parsons, G., Passini, M.A., et al. (2004). Intracerebral transplantation of adult mouse neural progenitor cells into the NiemannPick-A mouse leads to a marked decrease in lysosomal storage pathology. J. Neurosci. 24, 10642-10651.

Sim, F.J., Lang, J.K., Waldau, B., Roy, N.S., Schwartz, T.E., Pilcher, W.H., Chandross, K.J., Natesan, S., Merrill, J.E., and Goldman, S.A. (2006). Complementary patterns of gene expression by human oligodendrocyte progenitors and their environment predict determinants of progenitor maintenance and differentiation. Ann. Neurol. 59, 763-779.

Sim, F.J., Windrem, M.S., and Goldman, S.A. (2009). Fate determination of adult human glial progenitor cells. Neuron Glia Biol. 5, 45-55.

Sim, F.J., McClain, C.R., Schanz, S.J., Protack, T.L., Windrem, M.S., and Goldman, S.A. (2011). CD140a identifies a population of highly myelinogenic, migration-competent and efficiently engrafting human oligodendrocyte progenitor cells. Nat. Biotechnol. 29, 934-941.

Simon, K., Hennen, S., Merten, N., Blättermann, S., Gillard, M., Kostenis, E., and Gomeza, J. (2016). The Orphan G Protein-coupled Receptor GPR17 Negatively Regulates Oligodendrocyte Differentiation via Gai/o and Its Downstream Effector Molecules. J. Biol. Chem. 291, 705-718.

Smith, B.M., Giddens, M.M., Neil, J., Owino, S., Nguyen, T.T., Duong, D., Li, F., and Hall, R.A. (2017). Mice lacking Gpr37 exhibit decreased expression of the myelin-associated glycoprotein MAG and increased susceptibility to demyelination. Neuroscience 358, 49-57.

Soneson, C., Love, M.I., and Robinson, M.D. (2015). Differential analyses for RNA-seq: transcript-level estimates improve gene-level inferences. F1000Res. 4, 1521.

Stidworthy, M.F., Genoud, S., Suter, U., Mantei, N., and Franklin, R.J. (2003). Quantifying the early stages of remyelination following cuprizone-induced demyelination. Brain Pathol. 13, 329-339.

Tamaki, S.J., Jacobs, Y., Dohse, M., Capela, A., Cooper, J.D., Reitsma, M., He, D., Tushinski, R., Belichenko, P.V., Salehi, A., et al. (2009). Neuroprotection of host cells by human central nervous system stem cells in a mouse model of infantile neuronal ceroid lipofuscinosis. Cell Stem Cell 5, 310-319.

Tkachev, D., Mimmack, M.L., Ryan, M.M., Wayland, M., Freeman, T., Jones, P.B., Starkey, M., Webster, M.J., Yolken, R.H., and Bahn, S. (2003). 
Oligodendrocyte dysfunction in schizophrenia and bipolar disorder. Lancet 362, 798-805.

Tomaru, Y., Nakanishi, M., Miura, H., Kimura, Y., Ohkawa, H., Ohta, Y., Hayashizaki, Y., and Suzuki, M. (2009). Identification of an inter-transcription factor regulatory network in human hepatoma cells by Matrix RNAi. Nucleic Acids Res. 37, 1049-1060.

Wang, S., Bates, J., Li, X., Schanz, S., Chandler-Militello, D., Levine, C., Maherali, N., Studer, L., Hochedlinger, K., Windrem, M., and Goldman, S.A. (2013). Human iPSC-derived oligodendrocyte progenitor cells can myelinate and rescue a mouse model of congenital hypomyelination. Cell Stem Cell 12, 252-264.

Weng, Q., Chen, Y., Wang, H., Xu, X., Yang, B., He, Q., Shou, W., Chen, Y., Higashi, Y., van den Berghe, V., et al. (2012). Dual-mode modulation of Smad signaling by Smad-interacting protein Sip1 is required for myelination in the central nervous system. Neuron 73, 713-728.

Windrem, M.S., Roy, N.S., Wang, J., Nunes, M., Benraiss, A., Goodman, R., McKhann, G.M., 2nd, and Goldman, S.A. (2002). Progenitor cells derived from the adult human subcortical white matter disperse and differentiate as oligodendrocytes within demyelinated lesions of the rat brain. J. Neurosci. Res. 69, 966-975.

Windrem, M.S., Nunes, M.C., Rashbaum, W.K., Schwartz, T.H., Goodman, R.A., McKhann, G., 2nd, Roy, N.S., and Goldman, S.A. (2004). Fetal and adult human oligodendrocyte progenitor cell isolates myelinate the congenitally dysmyelinated brain. Nat. Med. 10, 93-97.

Windrem, M.S., Schanz, S.J., Guo, M., Tian, G.F., Washco, V., Stanwood, N., Rasband, M., Roy, N.S., Nedergaard, M., Havton, L.A., et al. (2008). Neonatal chimerization with human glial progenitor cells can both remyelinate and rescue the otherwise lethally hypomyelinated shiverer mouse. Cell Stem Cell 2, 553-565.

Windrem, M.S., Schanz, S.J., Morrow, C., Munir, J., Chandler-Militello, D., Wang, S., and Goldman, S.A. (2014). A competitive advantage by neonatally engrafted human glial progenitors yields mice whose brains are chimeric for human glia. J. Neurosci. 34, 16153-16161.
Xiang, X., Zhang, X., and Huang, Q.-L. (2012). Plexin A3 is involved in semaphorin 3F-mediated oligodendrocyte precursor cell migration. Neurosci. Lett. 530, 127-132.

Yandava, B.D., Billinghurst, L.L., and Snyder, E.Y. (1999). "Global" cell replacement is feasible via neural stem cell transplantation: evidence from the dysmyelinated shiverer mouse brain. Proc. Natl. Acad. Sci. USA 96 7029-7034.

Yang, H.-J., Vainshtein, A., Maik-Rachline, G., and Peles, E. (2016). G proteincoupled receptor 37 is a negative regulator of oligodendrocyte differentiation and myelination. Nat. Commun. 7, 10884.

Yoo, S.W., Motari, M.G., Susuki, K., Prendergast, J., Mountney, A., Hurtado, A., and Schnaar, R.L. (2015). Sialylation regulates brain structure and function. FASEB J. 29, 3040-3053.

Zaucker, A., Mercurio, S., Sternheim, N., Talbot, W.S., and Marlow, F.L. (2013). notch3 is essential for oligodendrocyte development and vascular integrity in zebrafish. Dis. Model. Mech. 6, 1246-1259.

Zhang, S.C., and Duncan, I.D. (2000). Remyelination and restoration of axonal function by glial cell transplantation. Prog. Brain Res. 127, 515-533.

Zhang, Y., Sloan, S.A., Clarke, L.E., Caneda, C., Plaza, C.A., Blumenthal, P.D. Vogel, H., Steinberg, G.K., Edwards, M.S., Li, G., et al. (2016). Purification and Characterization of Progenitor and Mature Human Astrocytes Reveals Transcriptional and Functional Differences with Mouse. Neuron 89, 37-53.

Zhao, C., Deng, Y., Liu, L., Yu, K., Zhang, L., Wang, H., He, X., Wang, J., Lu, C., Wu, L.N., et al. (2016). Dual regulatory switch through interactions of Tcf7l2/ Tcf4 with stage-specific partners propels oligodendroglial maturation. Nat. Commun. 7, 10883.

Zoupi, L., Savvaki, M., Kalemaki, K., Kalafatakis, I., Sidiropoulou, K., and Karagogeos, D. (2018). The function of contactin-2/TAG-1 in oligodendrocytes in health and demyelinating pathology. Glia 66, 576-591.

Zuchero, J.B., Fu, M.M., Sloan, S.A., Ibrahim, A., Olson, A., Zaremba, A., Dugas, J.C., Wienbar, S., Caprariello, A.V., Kantor, C., et al. (2015). CNS myelin wrapping is driven by actin disassembly. Dev. Cell 34, 152-167. 


\section{STAR $\star$ METHODS}

\section{KEY RESOURCES TABLE}

\begin{tabular}{|c|c|c|}
\hline REAGENT or RESOURCE & SOURCE & IDENTIFIER \\
\hline \multicolumn{3}{|l|}{ Antibodies used in tissue } \\
\hline Rat monoclonal anti-Myelin Basic Protein & Abcam & Cat\#ab7349; RRID: AB_305869 \\
\hline $\begin{array}{l}\text { Mouse monoclonal anti-human Nuclei, Clone } \\
235-1\end{array}$ & EMD Millipore & Cat\#MAB1281; RRID: AB_94090 \\
\hline $\begin{array}{l}\text { Mouse monoclonal anti-Human Cytoplasmic } \\
\text { Marker }\end{array}$ & Takara Bio Inc & Cat\#aB-121-U-050; RRID: AB_2632385 \\
\hline Mouse monoclonal anti-GFAP, clone SMI 21 & Covance Res. Products & Cat\#SMI-21R-500; RRID: AB_509979 \\
\hline Mouse anti-NG2, clone 9.2.27 & Millipore & Cat\# MAB2029; RRID: AB_94509 \\
\hline Rabbit anti-human PDGFRa, clone D13C6 & Cell Signal & Cat\#5241S; RRID: AB_10692773 \\
\hline Rabbit anti-Transferrin antibody (discontinued) & Abcam & Cat\#ab9538; RRID: AB_307325 \\
\hline Mouse anti-human Transferrin, clone HT1/13.6.3 & MP Biomedicals & Cat\#AM025; RRID: AB_10059965 \\
\hline Rabbit anti-CNP & Abcam & Cat\#18527; RRID: AB_470266 \\
\hline Mouse anti-human cytoplasmic antigen, STEM121 & Takara Bio & Cat\# AB-121-U-050; RRID:AB_2632385 \\
\hline Goat anti-mouse lgG $(\mathrm{H}+\mathrm{L})$ Alexa Fluor 647 & ThermoFisher & Cat\#A-21235; RRID: AB_2535804 \\
\hline Goat anti-mouse IgG1 Alexa Fluor 488 & ThermoFisher & Cat\#A-21121; RRID: AB_2535764 \\
\hline Goat anti-mouse IgG $(\mathrm{H}+\mathrm{L})$ Alexa Fluor 488 & ThermoFisher & Cat\#A-11029; RRID: AB_2534088 \\
\hline Goat anti-mouse IgG $(\mathrm{H}+\mathrm{L})$ Alexa Fluor 568 & ThermoFisher & Cat\#A-11031; RRID: AB_144696 \\
\hline Goat anti-mouse lgG1 Alexa Fluor 568 & ThermoFisher & Cat\#A-21124; RRID: AB_2535766 \\
\hline Goat anti-Rabbit IgG $(\mathrm{H}+\mathrm{L})$ Alexa Fluor 647 & ThermoFisher & Cat\#A-21245; RRID: AB_2535813 \\
\hline Goat anti-Rabbit IgG $(\mathrm{H}+\mathrm{L})$ Alexa Fluor 568 & ThermoFisher & Cat\#A-11036; RRID: AB_2534094 \\
\hline Goat anti-Rabbit IgG $(\mathrm{H}+\mathrm{L})$ Alexa Fluor 488 & ThermoFisher & Cat\#A-11034; RRID: AB_2576217 \\
\hline Goat anti-Rat IgG $(\mathrm{H}+\mathrm{L})$ Alexa Fluor 647 & ThermoFisher & Cat\#A-21247; RRID: AB_141778 \\
\hline Goat anti-Rat IgG $(\mathrm{H}+\mathrm{L})$ Alexa Fluor 568 & ThermoFisher & Cat\#A-11077; RRID: AB_2534121 \\
\hline Goat anti-Rat IgG $(\mathrm{H}+\mathrm{L})$ Alexa Fluor 488 & ThermoFisher & Cat\#A-11006; RRID: AB_2534074 \\
\hline \multicolumn{3}{|l|}{ Antibodies used in vitro } \\
\hline Mouse anti-PSA-NCAM, clone 2-2B & Millipore & Cat\#MAB5324; RRID: AB_95211 \\
\hline Mouse anti-PSA-NCAM supernatant, clone 5 A5 & DSHB & Cat\#5A5; RRID: AB_528392 \\
\hline Mouse anti-A2B5 supernatant, clone 105 & ATCC & Cat\#CRL-1520; RRID: CVCL_7946 \\
\hline Mouse anti-CD140a, clone AR1 & BD Biosci. & Cat\#556001; RRID: AB_396285 \\
\hline PE-conjugated mouse anti-human CD140a, AR1 & BD Biosci. & Cat\#556002; RRID: AB_396286 \\
\hline Rat anti-Mouse IgM MicroBeads & Miltenyi & $\begin{array}{l}\text { [REMOVED HYPERLINK FIELD]Cat\#130-047-301; } \\
\text { RRID: AB_244358 }\end{array}$ \\
\hline Rat anti-Mouse IgG2a+b MicroBeads & Miltenyi & Cat\# 130-047-201; RRID: AB_244356 \\
\hline \multicolumn{3}{|c|}{ Chemicals, Peptides, and Recombinant Proteins used in tissue processing } \\
\hline Phosphate Buffer Powder & Sigma-Aldrich & Cat\#P7994 \\
\hline Phosphate Buffer & Sigma-Aldrich & Cat\#P36919-1GA \\
\hline Thimerosal & Sigma-Aldrich & Cat\#T5125-10G \\
\hline Sodium Chloride & Fisher Scient. & Cat\#S271-3 \\
\hline Saponin & Sigma-Aldrich & Cat\#47036-50G-F \\
\hline Triton X-100 Surfactant & EMD Millipore & Cat\#9400-100ML \\
\hline Sucrose & Fisher Scient. & Cat\#50-230-0354 \\
\hline Paraformaldehyde & Fisher Scient. & Cat\#O4042-500 \\
\hline Sodium Hydroxide & EMD Millipore & Cat\#SX0607H-6 \\
\hline Hydrochloric Acid & Fisher Scient. & Cat\#A144-212 \\
\hline
\end{tabular}

(Continued on next page) 


\begin{tabular}{|c|c|c|}
\hline \multicolumn{3}{|l|}{ Continued } \\
\hline REAGENT or RESOURCE & SOURCE & IDENTIFIER \\
\hline 2-Methylbutane & EMD Millipore & Cat\#MX0760-1 \\
\hline O.C.T. Compound & Fisher Scient. & Cat\#14-373-65 \\
\hline Normal Goat Serum & ThermoFisher & Cat\#16210072 \\
\hline PIPES & Sigma & Cat\#P8658 \\
\hline L-cysteine-HCL & Sigma & Cat\#C7477-25 g \\
\hline $0.5 \mathrm{M}$ EDTA, pH8 & Invitrogen & Cat\#15575-020 \\
\hline DNasel & Sigma & Cat\#D4263-5VL \\
\hline 10X PBS, pH7.2 & Invitrogen & Cat\#70013-032 \\
\hline $0.5 \mathrm{M}$ EDTA, pH8 & Invitrogen & Cat\#15575-020 \\
\hline Liberase & Fisher Scientific & Cat\#NC9953702 \\
\hline Plasma-Derived FBS & Cocalico & Cat\#54-0115 \\
\hline \multicolumn{3}{|l|}{ Experimental Models: Organisms/Strains } \\
\hline Mouse: C3Fe.SWV-Mbpshi/J & Jackson Labs & Cat\#001428 \\
\hline Mouse: B6.129S7-Rag1tm1Mom/J & Jackson Labs & Cat\#002216 \\
\hline Mouse: C3H.129S6(B6)-Rag2tm1FwaN12 & Taconic & Cat\#000602-M; discont'd \\
\hline \multicolumn{3}{|l|}{ Software and Algorithms } \\
\hline Photoshop CS6 & Adobe & $\mathrm{N} / \mathrm{A}$ \\
\hline Illustrator CS6 & Adobe & $\mathrm{N} / \mathrm{A}$ \\
\hline Stereolnvestigator v11 & MBF Biosci. & $\mathrm{N} / \mathrm{A}$ \\
\hline Neurolucida Explorer v11 & MBF Biosci. & $N / A$ \\
\hline Leica Metamorph AF v2 & Leica Biosyst. & $\mathrm{N} / \mathrm{A}$ \\
\hline Leica Application Suite X & Leica Biosyst. & N/A \\
\hline Prism & GraphPad & $\mathrm{N} / \mathrm{A}$ \\
\hline Trimmomatic (version 0.32) & $N / A$ & $\begin{array}{l}\text { http://www.usadellab.org/cms/? } \\
\text { page=trimmomatic }\end{array}$ \\
\hline STAR (version 2.5.4b) & $\mathrm{N} / \mathrm{A}$ & https://github.com/alexdobin/STAR \\
\hline RSEM (version 1.3.1) & $\mathrm{N} / \mathrm{A}$ & https://deweylab.github.io/RSEM/ \\
\hline $\mathrm{R}$ & $\mathrm{N} / \mathrm{A}$ & https://www.R-project.org/ \\
\hline RUVSeq (version 1.6.2) & N/A & $\begin{array}{l}\text { http://www.bioconductor.org/packages/release/ } \\
\text { bioc/html/RUVSeq.html }\end{array}$ \\
\hline EDASeq (version 2.6.2) & N/A & $\begin{array}{l}\text { http://www.bioconductor.org/packages/release/ } \\
\text { bioc/html/EDASeq.html }\end{array}$ \\
\hline edgeR (version 3.14.0) & $N / A$ & $\begin{array}{l}\text { http://www.bioconductor.org/packages/release/ } \\
\text { bioc/html/edgeR.html }\end{array}$ \\
\hline DESeq2 (version 1.12.4) & N/A & $\begin{array}{l}\text { http://www.bioconductor.org/packages/release/ } \\
\text { bioc/html/DESeq2.html }\end{array}$ \\
\hline Tximport (version 1.8.0) & $N / A$ & $\begin{array}{l}\text { https://bioconductor.org/packages/release/bioc/ } \\
\text { html/tximport.html }\end{array}$ \\
\hline Readr (version 1.3.1) & $\mathrm{N} / \mathrm{A}$ & $\begin{array}{l}\text { https://cran.rstudio.com/web/packages/readr/ } \\
\text { index.html }\end{array}$ \\
\hline Gephi (version 0.9.1) & N/A & https://gephi.org/ \\
\hline Ingenuity Pathway Analysis & N/A & $\begin{array}{l}\text { https://digitalinsights.qiagen.com/ } \\
\text { products-overview/discovery-insights-portfolio/ } \\
\text { content-exploration-and-databases/qiagen-ipa/ }\end{array}$ \\
\hline pHeatmap (version 1.0.12) & $\mathrm{N} / \mathrm{A}$ & https://github.com/raivokolde/pheatmap \\
\hline ggplot2 (version 3.1.1) & $\mathrm{N} / \mathrm{A}$ & https://ggplot2.tidyverse.org/ \\
\hline Cytoscape & $\mathrm{N} / \mathrm{A}$ & https://cytoscape.org/ \\
\hline \multicolumn{3}{|l|}{ Other } \\
\hline Cryostat & Hacker Instruments & Model OTF \\
\hline Cryostat & Leica Biosyst. & Cat\#CM3050S \\
\hline
\end{tabular}




\begin{tabular}{|c|c|c|}
\hline \multicolumn{3}{|l|}{ Continued } \\
\hline REAGENT or RESOURCE & SOURCE & IDENTIFIER \\
\hline Disposable microtome blades & C.L. Sturkey & 1000 Plus \\
\hline Surgipath X-tra precleaned micro slides & Leica Biosyst. & Cat\#121-6 \\
\hline FlowView300 & Olympus & $\mathrm{N} / \mathrm{A}$ \\
\hline DMi8 & Leica Biosyst. & N/A \\
\hline DM6000B & Leica Biosyst. & N/A \\
\hline DFC 360 FX camera & Leica Biosyst. & $\mathrm{N} / \mathrm{A}$ \\
\hline BX51 & Olympus & $\mathrm{N} / \mathrm{A}$ \\
\hline DP30BW camera & Olympus & N/A \\
\hline Orca-R2 Digital CCD Camera & Hamamatsu & Cat\#C10600-10B \\
\hline MAC 5000 & Ludl Electronic & Cat\#73005001 \\
\hline Focus DR Linear encoder & Ludl Electronic & Cat\#99A420 \\
\hline STG 4"x3" Stepper & Ludl Electronic & Cat\#99S100-LE2-MBF \\
\hline Olympus IX71 Inverted Research Microscope & Olympus & N/A \\
\hline Agilent Bioanalyzer & Agilent & $\mathrm{N} / \mathrm{A}$ \\
\hline BD FACS AriallIU & BD Bioscience & $N / A$ \\
\hline HiSeq 2500 & Illumina Inc. & $\mathrm{N} / \mathrm{A}$ \\
\hline Nanodrop 1000 spectrophometer & Nanodrop & $\mathrm{N} / \mathrm{A}$ \\
\hline ClampFit & Axon Instr. & $N / A$ \\
\hline Multiclamp 700B amplifier & Axon Instr. & $\mathrm{N} / \mathrm{A}$ \\
\hline DigiData 1440 & Axon Instr. & N/A \\
\hline PC10 two-step electrode puller & Narishige & N/A \\
\hline DigiGait treadmill & Mouse Specifics Inc & $\mathrm{N} / \mathrm{A}$ \\
\hline Leica VT1000s & Leica Biosyst. & N/A \\
\hline \multicolumn{3}{|l|}{ Critical Commercial Assays } \\
\hline Ovation PicoSL WTA System V2 & NuGEN & Cat\#3312 \\
\hline Prelude Direct Lysis Modul & NuGen & Cat\#1400-24 \\
\hline \multicolumn{3}{|l|}{ Deposited Data } \\
\hline Raw RNA-seq data & GEO datasets & GEO: GSE112557 \\
\hline R Analysis Script and Processed data & This paper & $\begin{array}{l}\text { https://github.com/CTNGoldmanLab/ } \\
\text { Cuprizone_2020 }\end{array}$ \\
\hline
\end{tabular}

\section{RESOURCE AVAILABILITY}

\section{Lead Contact}

Further information and requests for resources and reagents should be directed to and will be fulfilled by the Lead Contact, Steven A. Goldman (steven_goldman@urmc.rochester.edu).

Materials availability

Any unique reagents generated in this study are available from the Lead Contact with a completed Materials Transfer Agreement.

Data and Code Availability

Raw gene expression data are available via GEO, accession number GSE112557.

Exploration of our gene expression data can be carried out at https://genial.is/cuprizone. Full R analysis script and all processed data are available at https://github.com/CTNGoldmanLab/Cuprizone_2020.

\section{EXPERIMENTAL MODEL AND SUBJECT DETAILS}

Animal models and transplantation

Shiverer mice, engrafted as adults

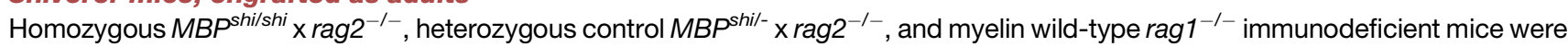
bred and housed in a pathogen-free environment in accordance with University of Rochester animal welfare regulations. Mice from 
each genotype were transplanted between the ages of 4-6 weeks with $1 \times 10^{5} \mathrm{hGPCs} / 1 \mu \mathrm{l} / \mathrm{hemisphere}(\mathrm{n}=2-4 \mathrm{mice} / \mathrm{time}-\mathrm{point} / \mathrm{ge}-$ notype), delivered bilaterally to the genu of the corpus callosum at coordinates: $A P-0.8 ; M L \pm 0.75$; $D V-1.25$, all relative to bregma. Mice were injected with FK506 (5 mg/kg, i.p,.; Tecoland) daily for 3 days pre- and 3 days post-surgery. All MBP ${ }^{\text {shi/shi }} \times$ Rag2 ${ }^{-1-}$ mice were killed at age 19-20 weeks, or when clinical morbidity, as defined in our animal welfare policy, was observed. For the myelin wildtype mice, half of all rag2 ${ }^{-/-}$and $\mathrm{rag}^{-/-}$animals were sacrificed between 19-21 weeks of age, and the other half at 1 year.

Myelin wild-type mice, neonatally transplanted, cuprizone-demyelinated as adults

Homozygous rag $1^{-1-}$ immunodeficient mice on a C57BL/6 background were bred in our colony. Animals were transplanted with hGPCs neonatally, via bilateral injections delivered to the presumptive corpus callosum (Windrem et al., 2004), so as to engraft newborn recipient brains before cuprizone demyelination. Beginning at 17 weeks of age, these mice were fed ad libitum a diet containing $0.2 \%(\mathrm{w} / \mathrm{w})$ cuprizone (S5891, BioServe) for 12 weeks and then returned to normal diet. Littermate and nonlittermate controls were maintained on a normal diet. Mice were sacrificed before diet (17 weeks), during diet ( 25 weeks), immediately after diet completion ( 29 weeks), and after either 8 weeks (37 weeks old) or 20 weeks (49 weeks old) of post-cuprizone recovery.

Myelin wild-type mice, cuprizone-demyelinated as adults, then transplanted

Homozygous rag $1^{-1-}$ mice were subjected to cuprizone demyelination as noted, for a 20 -week period beginning at 6 weeks of age. They were transplanted with hGPCs at 10 weeks of age, 4 weeks into their period of cuprizone demyelination. At that point, the mice were transplanted with a total of 200,000 PSA-NCAM $/$ A2B5 ${ }^{+}$cells, delivered sterotaxically as $1 \times 10^{5}$ hGPCs/1 $\mu$ l HBSS into the corpus callosum bilaterally at the following coordinates: from bregma, $A P-0.8 \mathrm{~mm}, \mathrm{ML} \pm 0.75$; from dura, DV $-1.25 \mathrm{~mm}$. Upon recovery, mice were returned to their cages. Mice were injected with FK506 ( $5 \mathrm{mg} / \mathrm{kg}$, i.p.; Tecoland) daily for 3 days pre- and 3 days post-surgery. Mice were sacrificed during diet (18 weeks), immediately after diet completion (26 weeks), and after 20 weeks (46 weeks old) of post-cuprizone recovery.

\section{Cells}

Human glial progenitor cells (hGPCs) were sorted from 18-22 week g.a. human fetuses, obtained from the surgical pathology suite, by

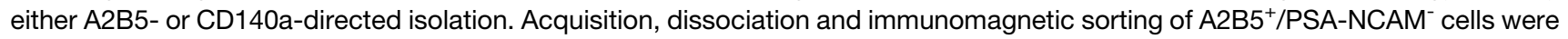
as described (Windrem et al., 2004). GPCs were isolated from dissociated tissue using a dual immunomagnetic sorting strategy: depleting mouse anti-PSA-NCAM ${ }^{+}$(Millipore, DSHB) cells, using microbead tagged rat anti-mouse IgM (Miltenyi Biotech), then selecting A2B5 ${ }^{+}$(clone 105; ATCC, Manassas, VA) cells from the PSA-NCAM- pool, as described (Windrem et al., 2004, 2008). After sorting, cells were maintained for 1-14 days in DMEM-F12/N1 with $10 \mathrm{ng} / \mathrm{ml} \mathrm{bFGF} \mathrm{and} 20 \mathrm{ng} / \mathrm{ml}$ PDGF-AA. Alternatively for some experiments, CD140a/PDGF $\alpha$ R-defined GPCs were isolated and sorted using MACS as described (Sim et al., 2011), yielding an enriched population of $\mathrm{CD} 140^{+}$glial progenitor cells.

\section{METHOD DETAILS}

\section{Quantitative histology}

All mice were perfused with calcium- and magnesium-free HBSS followed by $4 \%$ paraformaldehyde. Brains were cryopreserved with $6 \%$, then $30 \%$ sucrose and embedded coronally in OCT (TissueTek). Brains were then cut at $20 \mu \mathrm{m}$ on a Leica cryostat. Sections were immunolabled for one or more of the antigenic markers listed below. The optical fractionator method was used to quantify each cell phenotype in the corpus callosum, as: oligodendrocytes (transferrin), astrocytes (GFAP), and progenitors (PDGFR $\alpha$ ). Quantification was performed by using a computerized stereology system consisting of a BX-51 microscope (Olympus) equipped with a Ludl (Hawthorne, NY) XYZ motorized stage, Heidenhain (Plymouth, MN) z axis encoder, an Optronics (East Muskogee, OK) QuantiFire black and white video camera, a Dell (Round Rock, TX) PC workstation, and Stereo Investigator software (MicroBrightField, Wiliston, VT). Within each corpus callosum, beginning at a random starting point where it crosses the midline, 3 sections equidistantly spaced $480 \mu \mathrm{m}$ apart were selected for analysis. The corpus callosum was outlined from the midline to $1 \mathrm{~mm}$ lateral, and all cells were counted. Upper and lower exclusion zones of $10 \%$ of section thickness were used.

\section{Functional assessment}

Surgery

To assess the functional benefits effects of hGPC transplantation in young adult shiverer mice, 16 young adult shiverer $x$ rag 2 null mice (8 male, 8 female) underwent surgery after weaning, at 4 wks of age. Half received human fetal CD140a-sorted cells, and half received vehicle control. All mice were injected bilaterally at 4 points in the corpus callosum (AP: -0.8 or +0.9 , ML: $+/-0.75$ ). The bilateral rostral injections were extended $3.0 \mathrm{~mm}$ ventrally into the striatum, with stops for partial injection at $-3.0,-2.25$ and $-1.5 \mathrm{~mm}$, while the caudal injections were placed $1.25 \mathrm{~mm}$ ventral to the pial surface.

Motor and gait analysis

All 16 mice were tested individually on a DigiGait treadmill (Mouse Specifics, Inc., Framingham, MA). Treadmill speed for young, healthy mice is $16 \mathrm{~cm} / \mathrm{sec}$; for both transplanted and control 18-week old shiverers, the speed was set to $10 \mathrm{~cm} / \mathrm{sec}$. Videos were captured for $5 \mathrm{~s}$ periods, and the resultant images analyzed using DigiGait's incorporated gait analysis software. 


\section{Recording of transcallosal compound action potentials}

At 18-20 weeks of age, after their gait assessments, the mice were killed and coronal brain slices ( $350 \mu \mathrm{m})$ were prepared. Briefly, slices were cut using a Leica VT1000s (Leica Biosystems, Buffalo Grove, IL), into ice-cold buffer (in mM: $127 \mathrm{NaCl}_{2} 26 \mathrm{NaHCO}_{3}, 1.2$ $\mathrm{KH}_{2} \mathrm{PO}_{4}, 1.9 \mathrm{KCl}, 1.1 \mathrm{CaCl}_{2}, 2 \mathrm{MgSO}_{4}, 10 \mathrm{D}$-Glucose) bubbled with $95 \% \mathrm{O}_{2}$ and $5 \% \mathrm{CO}_{2}$. Slices were then transferred to a holding chamber containing oxygenated artificial cerebrospinal fluid (ACSF; in mM: $127 \mathrm{NaCl}, 26 \mathrm{NaHCO}_{3}, 1.2 \mathrm{KH}_{2} \mathrm{PO}_{4}, 1.9 \mathrm{KCl}_{2} .2 \mathrm{CaCl}_{2}, 1$ $\mathrm{MgSO}_{4}, 10 \mathrm{D}$-Glucose) for $30 \mathrm{~min}$ at $34^{\circ} \mathrm{C}$, for another $30 \mathrm{~min}$ at room temperature, and then transferred to a submersion recording chamber continually perfused with oxygenated ACSF at room temperature (rate: $2 \mathrm{ml} / \mathrm{min}$ ). Slices were equilibrated for at least $15 \mathrm{~min}$ before each recording.

Compound action potential recordings were made using a Multiclamp 700B amplifier (Axon Instruments, Foster City, CA), sampled at $10 \mathrm{kHz}$, digitized by a DigiData 1440A, and later analyzed offline by ClampFit (Axon software). Recording pipettes with resistances ranging between 3-6 $\mathrm{M} \Omega$ were pulled from standard borosilicate capillaries using a two-step electrode puller (PC-10, Narishige, Amityville, NY), and were filled with ACSF. A bipolar stimulating electrode and the recording electrode were placed on opposite sides of the corpus callosum, equidistant to the midline of each slice. The distance between stimulating electrode and recording electrode was between $1200-1600 \mu \mathrm{m}$. The first negative peak was measured as the myelinated component, and the second negative peak was measured as the unmyelinated component of CAPs.

Immunostaining

Phenotyping of donor cells was accomplished by immunostaining for human nuclear antigen (Millipore, clone MAB1281), together with one or more of the following, at the stated dilutions:

\begin{tabular}{|c|c|c|c|c|c|c|}
\hline & Antigen & Name & dilution & Catalog & Company & RRID \\
\hline \multirow[t]{8}{*}{ In vivo } & hGFAP & Mouse anti-human glial fibrillary acidic prot & $1: 500$ & SMI-21R & Covance & AB_509979 \\
\hline & $\mathrm{hN}$ & Mouse anti-human nuclei, clone $235-1$ & $1: 800$ & MAB1281 & Millipore & AB_94090 \\
\hline & hNG2 & Mouse anti-NG2, clone 9.2.27 & $1: 200$ & MAB2029 & Millipore & AB_94509 \\
\hline & MBP & Rat anti-myelin basic protein & $1: 25$ & ab7349 & Abcam & AB_305869 \\
\hline & hPDGFRa & Rabbit anti-human PDFaR, clone D13C6 & $1: 300$ & $5241 S$ & Cell Signal & AB_10692773 \\
\hline & Transferrin & Rabbit anti-human transferrin & $1: 800$ & ab9538 & Abcam & AB_307325 \\
\hline & CNP & Rabbit anti-CNP & $1: 400$ & 18527 & Abcam & AB_470266 \\
\hline & $\begin{array}{l}\text { Human } \\
\text { cytoplasm }\end{array}$ & $\begin{array}{l}\text { Mouse anti-human cytoplasmic antigen, } \\
\text { STEM121 }\end{array}$ & $1: 500$ & $A B-121 \cup 050$ & Takara Bio & AB_2632385 \\
\hline \multirow[t]{5}{*}{ In vitro } & PSA-NCAM & Mouse anti-PSA-NCAM, clone 2-2B & $1: 100$ & MAB5324 & Millipore & AB_95211 \\
\hline & PSA-NCAM & Mouse anti-PSA-NCAM supernatant, 5 A5 & $1: 1$ & $5 \mathrm{~A} 5$ & DSHB & AB_528392 \\
\hline & A2B5 & Mouse anti-A2B5 supernatant, clone 105 & $1: 1$ & CRL-1520 & ATCC & CVCL_7946 \\
\hline & CD140 & Mouse anti-CD140a, Clone AR1 & $0.21 \mu \mathrm{g} / 10^{6}$ cells & 556001 & BD Biosci. & AB_396285 \\
\hline & CD140a-PE & PE-conj mouse anti-human CD140a, AR1 & $10 \mu \mathrm{L} / 10^{6}$ cells & 556002 & BD Biosci & AB_396286 \\
\hline \multirow[t]{11}{*}{ Secondaries } & & Rat anti-Mouse IgM MicroBeads & $20 \mu \mathrm{L} / 10^{7}$ cells & $130-047-301$ & Miltenyi & AB_244358 \\
\hline & & Rat anti-Mouse IgG2a+b MicroBeads & $10 \mu \mathrm{L} / 10^{7}$ cells & $130-047-201$ & Miltenyi & AB_244356 \\
\hline & & AlexaFluor 568 Goat anti-Mouse $\lg G(H+L)$ & $1: 400$ & A-11031 & Invitrogen & AB_144696 \\
\hline & & AlexaFluor 568 Goat anti-Mouse IgG1 & $1: 400$ & A-21124 & Invitrogen & AB_2535766 \\
\hline & & AlexaFluor 488 Goat anti-Mouse $\lg G(H+L)$ & $1: 400$ & A-11029 & Invitrogen & AB_2534088 \\
\hline & & AlexaFluor 488 Goat anti-Mouse IgG1 & $1: 400$ & A-21121 & Invitrogen & AB_2535764 \\
\hline & & AlexaFluor 647 Goat anti-Mouse lgG $(\mathrm{H}+\mathrm{L})$ & $1: 400$ & A-21235 & Jackson & AB_2535813 \\
\hline & & AlexaFluor 568 Goat anti-Rabbit lgG $(\mathrm{H}+\mathrm{L})$ & $1: 400$ & A-11036 & Invitrogen & AB_2534094 \\
\hline & & AlexaFluor 488 Goat anti-Rabbit lgG $(\mathrm{H}+\mathrm{L})$ & $1: 400$ & A-11034 & Invitrogen & AB_2576217 \\
\hline & & AlexaFluor 568 Goat anti-Rat IgG $(\mathrm{H}+\mathrm{L})$ & $1: 400$ & A-11077 & Invitrogen & AB_2534121 \\
\hline & & AlexaFluor 488 Goat anti-Rat IgG $(\mathrm{H}+\mathrm{L})$ & $1: 400$ & $A-11006$ & Invitrogen & AB_2534074 \\
\hline
\end{tabular}

\section{QUANTIFICATION AND STATISTICAL ANALYSIS}

\section{Statistics}

Statistics were performed using GraphPad Prism. Where variances were equal and data normally distributed, we used one-way analysis of variance (ANOVA); when unequal variances obtained, Kruskal-Wallis non-parametric analysis was used. All graphs are shown as means \pm SEM. 
RNA-Sequencing and analysis

To observe transcriptional changes in hGPCs following demyelination, neonatal rag $1^{-1-}$ mice were transplanted with $2 \times 10^{5} \mathrm{hGPCs}$ as described above. At 12 weeks, mice either maintained a normal diet or were transitioned to a $0.2 \%$ (w/w) cuprizone diet fed ad libitum until 24 weeks of age when they were returned to a normal diet. At 36 weeks, all mice were anesthetized with sodium pentobarbital, perfused with ice cold $\mathrm{HBSS}^{+/+}$(containing calcium and magnesium) (Thermo Fisher), and the brains extracted and placed into fresh $\mathrm{HBSS}^{+/+}$in a tissue culture plate. Excess $\mathrm{HBSS}^{+/+}$was removed and the corpus callosum of each animal was isolated surgically, minced, and suspended in $\mathrm{HBSS}^{-1-}$. Corpora callosa from two mice from the same experimental group were pooled for each study. The tissue was transferred to a $15 \mathrm{~mL}$ conical tube and rinsed twice with $\mathrm{HBSS}^{-1-}$. The tube was filled to maximum volume with HBSS $-/-$, and then centrifuged for $5 \mathrm{~min}$ at 1,500 rpm. The supernatant was then removed and 0.62 units Research Grade Liberase $\mathrm{DH}$ (Roche) was added, and the samples then incubated at $37^{\circ} \mathrm{C}$ for $40 \mathrm{~min}$ with gentle rocking. Following Liberase dissociation, $2 \mathrm{~mL}$ 0.5\% BSA in MEM (Thermo Fisher) supplemented with 500 units bovine pancreas DNase (Sigma) and $0.5 \mathrm{~mL}$ PD-FBS (Cocalico Biologicals) was added to the sample. The sample was triturated with a 1000 Pipetman and then passed through a $70 \mu \mathrm{m}$ cell strainer. MEM containing $0.5 \%$ BSA was then added to the tube to bring it to full volume, and the tube centrifuged at 1,500 rpm for $10 \mathrm{~min}$. Dissociated cells were then tagged with anti-CD140a-PE and sorted via FACS as previously reported (Sim et al., 2011). Cells were lysed and prepared for library construction via Prelude Direct Lysis Module (NuGEN) according to the manufacturer's protocol.

Libraries were constructed using Ovation RNA-Seq System V2 (NuGEN) according to manufacturer's protocol and sequenced with a read length of paired-end 125bp on a HiSeq 2500 system (Illumina). Reads were demultiplexed and cleaned using Trimmomatic (Bolger et al., 2014). Reads were aligned to human genome GRCh38,p10 and mapped to Ensembl reference 91 via STAR 2.5.2b (Dobin et al., 2013), with quantMode set to TranscriptomeSAM. Gene abundances and expected counts were then calculated using RSEM 1.3.0 (Li and Dewey, 2011). Expected counts were imported into $\mathrm{R}$ via tximport for differential expression analysis between cuprizone and control hGPCs (R Core Team, 2017; Soneson et al., 2015). Low-expressing genes were removed prior to analysis if their expected counts fell below a median of 3 in both conditions. Full within-lane normalization of samples was conducted using EDASeq to adjust for GC-content effects prior to the generation of library size adjusted counts via DESeq2 (Love et al., 2014 ; Risso et al., 2011). Differential expression between post-cuprizone and control hGPCs was then determined using DESeq2 following the addition of a variance factor to the generalized linear model. This factor was calculated using RUVSeq's RUVs function with all genes set as in silico negative controls (Risso et al., 2014). Genes with an adjusted $p$ value $<0.05$ were considered significant. Only genes with mean transcripts per million (TPM) $>5$ in either group were kept for functional analysis, as they were more likely to be biologically significant.

For functional analysis and inference of gene interactivity, a gene ontology network was constructed. Differentially expressed genes between both groups were analyzed in Ingenuity Pathway Analysis (QIAGEN) where 43 significantly enriched terms were selected based on relevance, along with their contributing differentially expressed genes, to be used as nodes. Along with the undirected edges derived from gene-GO term associations, edges were further generated via IPA's curated database connecting genes with known interactions. Network visualization was carried out in Cytoscape (Shannon et al., 2003) with the determination of modularity occurring in Gephi (Bastian et al., 2009). Nodes were clustered within their respective modules and aesthetically repositioned slightly. 